\title{
Anterograde Signaling by Nitric Oxide: Characterization and In Vitro Reconstitution of an Identified Nitrergic Synapse
}

\author{
Ji-Ho Park, Volko A. Straub, and Michael O'Shea \\ Sussex Centre for Neuroscience, School of Biological Sciences, University of Sussex, Brighton, East Sussex, BN1 9QG, \\ United Kingdom
}

\begin{abstract}
Nitric oxide (NO) is recognized as a signaling molecule in the CNS where it is a candidate retrograde neurotransmitter. Here we provide direct evidence that NO mediates slow excitatory anterograde transmission between the NO synthase (NOS)expressing B2 neuron and an NO-responsive follower neuron named B7nor. Both are motoneurons located in the buccal ganglia of the snail Lymnaea stagnalis where they participate in feeding behavior. Transmission between B2 and B7nor is blocked by inhibiting NOS and is suppressed by extracellular scavenging of NO. Furthermore, focal application of $\mathrm{NO}$ to the cell body of the B7nor neuron causes a depolarization that mimics the effect of B2 activity. The slow interaction between
\end{abstract}

the $\mathrm{B} 2$ and B7nor neurons can be re-established when the two neurons are cocultured, and it shows the same susceptibility to NOS inhibition and NO scavenging. In cell culture we have also examined spatial aspects of NO signaling. We show that before the formation of an anatomical connection, the presynaptic neuron can cause depolarizing potentials in the follower neuron at distances up to $50 \mu \mathrm{m}$. The strength of the interaction increases when the distance between the cells is reduced. Our results suggest that $\mathrm{NO}$ can function as both a synaptic and a nonsynaptic signaling molecule.

Key words: nitric oxide; Lymnaea; feeding behavior; nitrergic synapse; nonsynaptic; Aplysia
The discovery that nitric oxide (NO) is a signaling molecule in the nervous system (Garthwaite et al., 1988) opened new and unexpected dimensions in our thinking about how information is transmitted by neurons (Dawson and Snyder, 1994; Gelperin, 1994; Garthwaite and Boulton, 1995; O'Shea et al., 1998). For example, traditionally neurotransmission is thought to be spatially restricted and in the anterograde direction, that is, from the presynaptic to the postsynaptic neuron. However, the synthesis and release of NO do not require the subcellular machinery normally associated with the presynaptic terminal (Bredt and Snyder, 1992), and NO can diffuse through cell membranes (Lancaster, 1994; Wood and Garthwaite, 1994). NO may therefore act without the need for conventional synaptic connectivity, and its action may not be confined locally to the postsynaptic neuron (Hartell, 1996). Indeed in systems in which the postsynaptic neuron seems to influence the presynaptic neuron, the unconventional features of NO signaling make NO an attractive candidate retrograde transmitter (Hölscher, 1997).

Whether NO can function more conventionally as an anterograde transmitter in the CNS has received far less attention, even though transmission in the forward direction is suggested by the depolarizing responses to NO by neurons in the paraventricular nucleus (Bains and Ferguson, 1997). Because the synthesis of NO by the neuronal isoform of NO synthase (NOS) is regulated by calcium and calmodulin (Bredt and Snyder, 1990), there is no

Received March 11, 1998; revised April 24, 1998; accepted April 30, 1998.

This work was supported by the Biotechnology and Biological Sciences Research Council Grant IR3521-1; we would like to thank Gottlieb Daimler and Karl BenzStiflung for supporting V.A.S. We are grateful to George Kemenes and Paul Benjamin for their input throughout the prosecution of this research and for their useful comments on the manuscript.

Correspondence should be addressed to Dr. Michael O'Shea, Sussex Centre for Neuroscience, School of Biological Sciences, University of Sussex, Brighton, East Sussex, BN1 9QG, UK.

Copyright (C) 1998 Society for Neuroscience $\quad 0270-6474 / 98 / 185463-14 \$ 05.00 / 0$ reason why the release of NO should not be coupled to presynaptic electrical activity. In this respect, NO may function as a signaling molecule in a way that is comparable with a classical anterograde chemical transmitter.

Assessing the transmitter status of NO in the mammalian CNS is difficult in part because of the difficulty of recording simultaneously pre- and postsynaptically from identified neurons in the intact nervous system. In invertebrates, however, and particularly among molluscan model systems, this problem can be overcome by exploiting the advantages of the presence of large and uniquely identified neurons in the CNS. Using this approach, Jacklet (1995) reported the first evidence that NO is used as an excitatory anterograde transmitter at an identified synapse for the marine gastropod mollusc Aplysia. In this particular example, the identified presynaptic neuron is histaminergic (Weiss et al., 1986), indicating a cotransmitter role for NO. The role of NO in the molluscan CNS can also be conveniently studied at a systems level, where, for example, it has been shown to have a role in olfactory processing (Gelperin, 1994), the activation of feeding behavior (Elphick et al., 1995), and learning (Teyke, 1996).

In this paper we show that a previously identified NOSexpressing neuron called B2 (Moroz et al., 1994a,b) in the gastropod Lymnaea stagnalis releases NO when depolarized and evokes a NO-mediated slow depolarizing potential in an identified follower neuron that we name B7nor. We also show that the NO-activated B7nor neuron is coupled to the central pattern generator (CPG) for feeding behavior, and its pattern of activity shows that it participates in this behavior. Our previous work showed that NO is a transmitter of sensory neurons projecting from the lips to the CNS and that NO mediates the sensory activation of the feeding CPG (Elphick et al., 1995). The present findings extend the role of NO in feeding behavior by showing that NO is an excitatory transmitter between feeding neurons within the buccal ganglia, the principal central location for the 
neural circuit controlling this behavior (Rose and Benjamin, 1979). In experiments in which the two identified neurons are cocultured, we show that the nitrergic connection can faithfully be reconstituted in cell culture. Using the cell culture system, we verify that NO can function monosynaptically as an excitatory anterograde transmitter and provide evidence that it can influence targets nonsynaptically at distances up to $50 \mu \mathrm{m}$. Together these experiments suggest a role for NO as an excitatory transmitter in the feeding system of Lymnaea and indicate that NO can perform both synaptic and nonsynaptic functions in the CNS.

\section{MATERIALS AND METHODS}

Specimens of the pond snail Lymnaea stagnalis were supplied by Blades Biological (Kent, UK) and were fed on lettuce. All chemicals were purchased from Sigma (Sigma, Poole, UK) unless otherwise stated. Middle-size animals $(\sim 3 \mathrm{gm})$ were used for the experiments and were dissected in standard snail physiological saline buffered to pH 7.9 (Benjamin and Winlow, 1981).

Histochemistry and anatomy. A modified NADPH-diaphorase staining method was used (Grozdanovic et al., 1995). Tissue was fixed for $1 \mathrm{hr}$ at $4^{\circ} \mathrm{C}$ with $0.5 \%$ paraformaldehyde and $0.5 \%$ Triton X-100 in $0.1 \mathrm{M}$ PBS, $\mathrm{pH}$ 7.6. After several washes in $0.1 \mathrm{M}$ PBS, the staining procedure was performed with nitro blue tetrazolium $(0.5 \mathrm{mg} / \mathrm{ml})$ and $\beta$-NADPH $(1$ $\mathrm{mg} / \mathrm{ml}$ ) in $0.1 \mathrm{M}$ PBS. The staining proceeded for $45-60 \mathrm{~min}$ in the dark at room temperature. Tissue was dehydrated in ethanol, cleared with histoclear (National Diagnostics, Hessle, UK) for $1 \mathrm{hr}$, mounted in histomount (Nationa1 Diagnostics), and photographed.

To reveal simultaneously the arborizations of the two identified neurons, we used two fluorescent dyes with easily distinguished emission spectra, 5(6)-carboxy fluorescein at $520 \mathrm{~nm}$ (5-CF; 5\% in water; Eastman Kodak, Rochester, NY) and methoxypyrenetrisulfonic acid at $423 \mathrm{~nm}$ (MPTS; 20\% in water; Molecular Probes, Eugene, OR), an analog of cascade blue. The dyes were injected via an intracellular microelectrode into the neuronal soma using negative current pulses $(0.8 \mathrm{sec} ; 1 \mathrm{~Hz}$ for 20 min). Usually the B2 neuron was injected with MPTS, and the B7nor neuron was injected with 5-CF. After the injection, the preparation was soaked in $50 \%$ glycerol saline solution, mounted on a cavity slide, and examined with a fluorescence microscope with the appropriate excitation and transmission filters. This double dye-filling technique was successfully used previously to detect potential sites of synaptic contact between neurons of the Lymnaea feeding system (Staras et al., 1998).

Electrophysiology and pharmacology. Standard techniques developed for intracellular recording and stimulation of molluscan neurons were used. Intracellular penetration was facilitated by treating the preparation with a protease solution (protease $\mathrm{E} ; 1 \mathrm{mg} / \mathrm{ml}$ in saline solution) for 3-5 min. The CNS was continuously superfused with fresh physiological saline at $3 \mathrm{ml} / \mathrm{min}$ during the experiments (Park et al., 1995). To help distinguish between monosynaptic and polysynaptic connections, a modified saline that contained elevated $\mathrm{Mg}^{2+}$ and $\mathrm{Ca}^{2+}$ levels at four and seven times, respectively, their concentration in physiological saline was used (Getting, 1981; Winlow, 1987). The osmolarity of this high divalent cation saline (HiDi saline) was adjusted by reducing the concentration of sodium and adding $8 \mathrm{~mm}$ sucrose. For recording and analyzing the data, a personal computer (PC) and analog-to-digital interface system were used (CED 1401 plus Spike 2 for Windows; Cambridge Electronic Design, Cambridge, UK). Electrophysiological techniques used in the experiments in cell culture are given below.

For interference with the NO signaling pathway, the NOS inhibitors $N^{\omega}$-nitro-L-arginine (L-NNA) and $N^{\omega}$-nitro-L-arginine methyl ester (LNAME) were used at $1 \mathrm{~mm}$ (Hibbs et al., 1987). The inactive D-isomer was used in control experiments to test for the specificity of the inhibitory effects of L-NAME. In addition, to remove NO after its release, we applied the scavenger 2-phenyl-4,4,5,5-tetramethylimidazoline-1-oxyl-3oxide (PTIO; Calbiochem, La Jolla, CA) at $0.25 \mathrm{~mm}$ (Li et al., 1995; Dobashi et al., 1997). All solutions were made up in physiological saline and constantly superfused at $3 \mathrm{ml} / \mathrm{min}$ during the experiments unless otherwise stated. These compounds are without significant effect on electrophysiological parameters of either B2 or B7nor neurons (membrane potential and excitability).

Microfocal application of NO to neuronal cell bodies was achieved using a pressure ejection system (General Valve, Fairfield, NJ) connected to a micropipette containing NO-saturated saline, the NO donor sodium nitrocysteine (SNC), an alternative NO donor $S$-nitroso- $N$-acetyl peniz- ilamine (SNAP), the related control compound $N$-acetyl penizilamine (NAP), or control saline. The NO-saline was prepared by bubbling NO gas through degassed and nitrogen-purged physiological saline. The maximum saturated concentration of $\mathrm{NO}$ in aqueous solution at $20^{\circ} \mathrm{C}$ and at a partial pressure of one atmosphere is 2 mM (Kogan et al., 1963). A solution of the NO donor SNC was prepared according to the protocol of Lei et al. (1992). The SNC concentration before being ejected from the micropipette was $100 \mathrm{~mm}$. For control experiments, the SNC solution was degassed by exposing it to the air at room temperature for $1 \mathrm{~d}$, followed by purging it with nitrogen for $\sim 10 \mathrm{~min}$. The $\mathrm{pH}$ of the SNC and degassed SNC solution remained unaltered. SNAP and NAP were dissolved in saline and pressure ejected at a pipette concentration of $50 \mathrm{~mm}$. These compounds were also focally applied to cultured neurons (see below). For experiments on the intact CNS, the superfusion flow rate was increased to $5 \mathrm{ml} / \mathrm{min}$ to restrict the effect of the ejected NOcontaining solutions to a small volume close to the tip of the micropipette. SNC was ejected using single 10 psi pressure pulses of $20 \mathrm{msec}$ duration. SNAP and NAP were ejected with $40 \mathrm{msec}$ pressure pulses at 6 psi. The saturated NO-saline was ejected with trains of 10 psi pressure pulses of $200 \mathrm{msec}$ duration at $1 \mathrm{~Hz}$.

Because the B2 neuron is known to contain acetylcholine (Perry et al., 1998) and the peptides small cardioactive peptide (SCP) and myomodulin (Santama et al., 1994; Perry et al., 1998), we have investigated the possibility that the depolarization in B7nor is caused by these putative transmitters. To investigate cholinergic transmission, we bath applied hexamethonium chloride at $0.5 \mathrm{~mm}(n=4)$, five times the concentration known to block excitatory cholinergic transmission in the snail CNS (Yeoman et al., 1993). Cholinergic transmission was also investigated in the cell culture system, and details of the methods used for this are provided below. The myomodulin and SCP peptides were focally applied by pressure ejecting them from pipettes placed above the target cell bodies. The pipette concentration for myomodulin (myomodulin A) was $0.1 \mathrm{mM}$. The effects of SCP were studied by applying an equimolar mixture of $\mathrm{SCP}_{\mathrm{A}}$ and $\mathrm{SCP}_{\mathrm{B}}$, both at pipette concentrations of $0.1 \mathrm{~mm}$.

Isolation and culture of identified neurons. Identified neurons were marked for subsequent isolation and transfer into cell culture by the iontophoretic injection of the vital dye fast green $(0.1 \%$ in distilled water) into the soma. The labeling of neurons identified according to the criteria established for the identification of the B7nor neuron (see Results) ensured that the same neuron that was investigated in the intact CNS was transferred to the culture dish. The cell culture procedure was modified after the protocol of Ridgeway et al. (1991). Media used included normal saline (NS), antibiotic saline (ABS), defined medium (DM), and conditioned medium (CM). Normal saline contained $\mathrm{NaCl}(50 \mathrm{mM}), \mathrm{KCl}(1.6$ $\mathrm{mM}), \mathrm{MgCl}_{2}(3.5 \mathrm{mM}), \mathrm{CaCl}_{2}(2.0 \mathrm{~mm})$, and HEPES $(10 \mathrm{~mm})$ at a $\mathrm{pH}$ of 7.9 in culture grade water, whereas ABS also contained gentamycin (150 $\mu \mathrm{g} / \mathrm{ml}$ ). DM was made by mixing $100 \mathrm{ml}$ of special L-15 medium (Gibco, BRL, Paisley, UK), $80 \mathrm{ml}$ of NS, and $120 \mathrm{ml}$ of culture grade water and by adding glutamine $(30 \mathrm{mg})$, glucose $(16.2 \mathrm{mg})$, and gentamycin $(600 \mu \mathrm{l}$ of $10 \mathrm{mg} / \mathrm{ml}$ stock) to the solution. For the CM preparation, isolated brains that had been washed extensively in ABS were incubated in DM (two brains $/ \mathrm{ml}$ ). After $3 \mathrm{~d}$ of incubation, the $\mathrm{CM}$ was sterile filtered (Millex-GV $0.22 \mu \mathrm{m}$; Millipore, Bedford, MA). Aliquots of CM (1 ml) were directly pipetted onto culture dishes (Falcon 3001; Becton Dickinson, Rutherford, NJ) coated with poly-L-lysine (15-30 kDa; $1 \mathrm{mg} / \mathrm{ml}$ in 15 $\mathrm{mM}$ Tris buffer), and equal amounts of DM were added. The culture dishes were stored at $-20^{\circ} \mathrm{C}$ and thawed $2-3 \mathrm{hr}$ before their use.

After electrophysiological identification and intracellular labeling of B7nor neurons with fast green (see above), the isolated CNS was incubated in a mixture of trypsin $(0.67 \mathrm{mg} / \mathrm{ml})$ and collagenase/dispase $(1.33$ $\mathrm{mg} / \mathrm{ml}$; Boehringer Mannheim, Indianapolis, IN) in DM for $30 \mathrm{~min}$ at room temperature. The enzyme treatment was followed by a $10 \mathrm{~min}$ incubation in soybean trypsin inhibitor $(1 \mathrm{mg} / \mathrm{ml}$ in DM). The CNS was then pinned out in a dissection dish filled with high-osmolarity DM (30 $\mathrm{mM}$ glucose in DM). B2 and B7nor neurons were visually identified according to their intracellular label (B7nor) or their size and characteristic position (B2). Their cell bodies were exposed by mechanically disrupting the inner connective tissue. The cell bodies together with their main processes were isolated from the buccal ganglia by gentle suction with a fire-polished micropipette (tip diameter, $\sim 100 \mu \mathrm{m}$ ) prepared from $1.5 \mathrm{~mm}$ glass tubing (GC150T-10; Clark Electromedical Instruments, Pangbourne, UK) that had been coated with Sigmacote. After isolation, neurons were transferred onto culture dishes and cultured at $20^{\circ} \mathrm{C}$ for up to $5 \mathrm{~d}$.

Electrophysiological and pharmacological studies on cultured neurons. 
Culture dishes containing pairs of B2 and B7nor neurons that had grown extensive overlapping processes in cell culture were perfused with NS at a flow rate of $1-2 \mathrm{ml} / \mathrm{min}$ for at least $30 \mathrm{~min}$ before the experiment to remove all culture medium. The perfusion was maintained throughout the experiment. Cell bodies were impaled with microelectrodes filled with saturated potassium sulfate (tip resistance, 20-30 M $\Omega$ ), and pairs of neurons were recorded simultaneously. Records were acquired directly onto a PC or were stored on DAT tapes. All pairs of cells were tested for electrical coupling by injecting negative current pulses in the range from $-0.2 \mathrm{nA}$ to $-0.6 \mathrm{nA}$ in either of the two neurons. To interfere with the NO signaling pathway, the NOS inhibitor L-NAME and NO scavenger PTIO were dissolved in physiological saline and added to the perfusion system. The D-isoform of L-NAME, D-NAME, was used in control experiments.

To investigate cholinergic transmission in cell culture, we perfused D-tubocurarine (d-TC), an antagonist known to block both inhibitory and excitatory cholinergic transmission in the snail (Yeoman et al., 1993), through the culture dish at $0.1 \mathrm{~mm}$. We also tested for direct effects of $\mathrm{ACh}$ and $\mathrm{NO}$ on isolated B7nor neurons by focally applying either ACh ( 1 and $10 \mu \mathrm{M}$ ) or the NO donor SNAP (pipette concentration, $1 \mathrm{~mm}$ ). The related compound NAP was also applied in control experiments to check for unspecific effects of the NO donor SNAP.

\section{RESULTS}

\section{A NOS-expressing neuron (B2) evokes slow depolarization in an identified follower neuron (B7nor)}

The paired left and right B2 cells in the buccal ganglia of Lymnaea stagnalis are large, conspicuous, and well characterized neurons (Benjamin and Rose, 1979; Benjamin et al., 1979; Perry et al., 1998). They can be uniquely identified on the basis of the size (50-70 $\mu \mathrm{m}$ in diameter) and position of their cell bodies that lie on the dorsal surface at the anterior margin of the buccal ganglia. The expression of NOS by the B2 neurons was first reported by Moroz et al. (1994a,b), who showed that they are stained by the NOS-selective NADPH-diaphorase histochemical method and immunolabeled by an antibody to the neuronal isoform of NOS. We have verified these findings and show in Figure $1 A$ that $\mathrm{B} 2$ neurons are strongly diaphorase-positive in whole-mount preparations of the buccal ganglia.

A systematic search for neurons that respond to electrical activity in B2 resulted in the identification of a neuron, which we call B7nor, on the dorsal surface of the buccal ganglion with a cell body $\sim 20 \mu \mathrm{m}$ in diameter. Importantly this neuron was found to receive a slow depolarizing potential when the ipsilateral B2 neuron is electrically stimulated. The cell body of the B7nor neuron lies close to that of $\mathrm{B} 2$, but the position is not fixed between individuals and alone does not provide for unambiguous identification. Moreover there are a number of cell bodies of a similar size in the vicinity that do not respond to B2 stimulation. The morphological features of the B7nor neuron were revealed by intracellular injection with either 5-CF or MPTS (Fig. 1B,C). This reveals a consistent trajectory of an axon that projects ventrally and then loops laterally and anteriorly before exiting the buccal ganglion in the ipsilateral posterior jugalis nerve that innervates the posterior jugalis muscle that forms part of the muscular feeding apparatus known as the buccal mass (Benjamin and Rose, 1979). Double labeling $(n=21)$ was used to reveal the morphological relationship between the two neurons (Fig. 1D). It shows the expected morphology of the B2 neuron with the main axon projecting to the dorsobuccal nerve and some arborization extending toward the midline of the buccal ganglia (Perry et al., 1998). An arborizing branch of the axon of the B7nor neuron also projects toward the midline, forming an overlapping field with B2 processes (Fig. 1B).

The pattern of activity seen in B7nor (see below) defines a class of feeding motoneurons known as the B7 group that innervate the
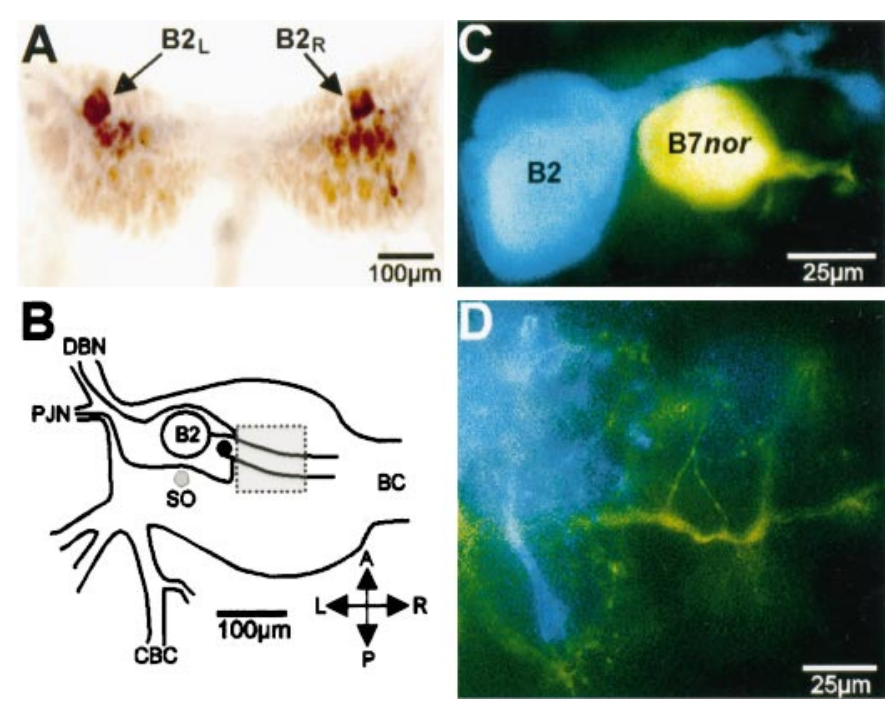

Figure 1. Anatomy of $\mathrm{B} 2$ and $\mathrm{B} 7$ nor neurons in the buccal ganglia. $A$, NADPH-diaphorase staining of the buccal ganglia. The pair of large (cell body, $\sim 50 \mu \mathrm{m}$ in diameter) diaphorase-positive cell bodies (arrows) are the left and right $\mathrm{B} 2$ neurons $\left(B 2_{L}, B 2_{R}\right)$, identified as NOS-expressing by Moroz et al. (1994a,b). There are also other unidentified positively stained neurons in the ganglia. $B$, A schematic diagram of the buccal ganglia and the positions of the cell bodies of neurons referred to in this article together with their main projections. The main axon branch of B2 leaves the ganglion via the dorsobuccal nerve $(D B N)$, whereas the axon of the B7nor neuron (cell body, filled circle) projects to the posterior jugalis nerve $(P J N)$. The overlapping aborizations shown by the double-dye fills in $D$ are near the midline of the ganglion in the area covered by the shaded rectangle. $A$, Anterior; $B C$, buccal commissure; $C B C$, cerebral-buccal connective; $P$, posterior; and $S O$, slow oscillator. $C$, Double-dye injection of the B2 and the identified follower neuron B7nor. In this experiment, the B2 neuron was injected with MPTS (blue), and the B7nor neuron was injected with 5-CF (yellow). Here the two cell bodies are apposed, but the position of the B7nor neuron is quite variable and can lie as much as 100 $\mu \mathrm{m}$ from the presynaptic B2 cell body. $D$, Detail of the overlapping fields of arborization of the B2 (blue) and B7nor (yellow) neurons whose cell bodies are shown in $C$.

posterior jugalis muscle (Benjamin and Rose, 1979; Rose and Benjamin 1979; Staras et al., 1998). The neuron we have identified is therefore by definition a type of B7 neuron. However, in two important respects, it is unlike other recognized B7 neurons. First, previously identified B7 neurons do not respond to B2 stimulation, and second, other B7 neurons innervate the jugalis muscle via the latero- or the ventrobuccal nerve. In contrast, the neuron we have identified receives slow excitatory input from B2 and exits the buccal ganglion via the posterior jugalis nerve. We named it B7nor to distinguish this neuron from other B7 neurons, indicating that it is nitric oxide responsive (justified below).

Single action potentials in B2 generally do not evoke unitary EPSPs in the B7nor neuron, but a brief burst of three or more action potentials at $\sim 10 \mathrm{~Hz}$ and above causes a smooth EPSP in B7nor with a delay of $\sim 200 \mathrm{msec}$ that in some preparations can reach spiking threshold (Fig. $2 A$ ). When the CNS is bathed in saline containing elevated concentrations of $\mathrm{Ca}^{2+}$ and $\mathrm{Mg}^{2+}$ (HiDi saline; $n=3$ ), unitary synaptic events can be recorded in the B7nor neuron (Fig. 2B). The use of HiDi saline in molluscan preparations is an accepted method for providing evidence to distinguish between mono- and polysynaptic pathways (Getting, 1981; Winlow, 1987). As spiking threshold is increased by HiDi saline, the probability that transmission through polysynaptic pathways will fail is increased. Because transmission persists 
A

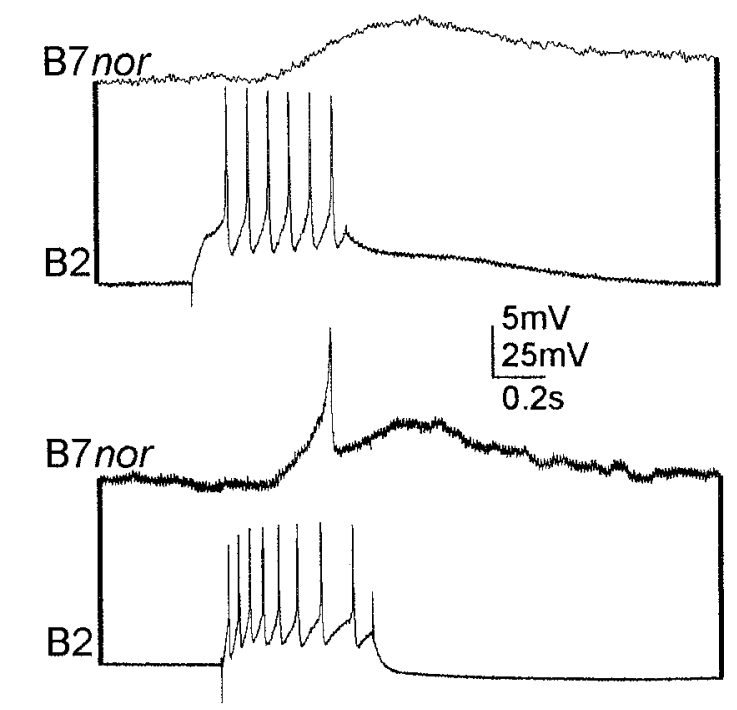

B

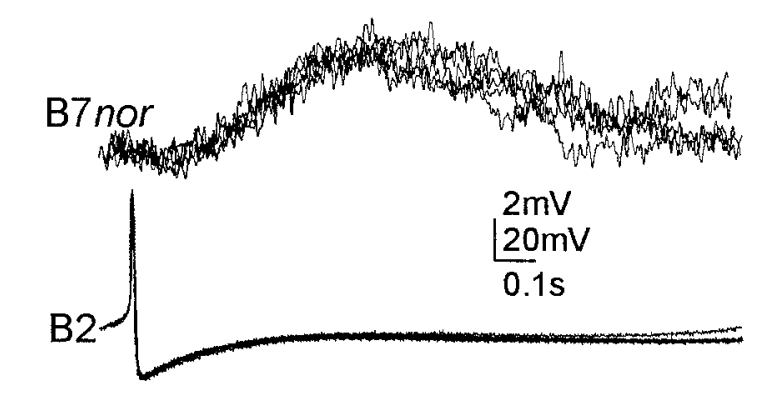

C

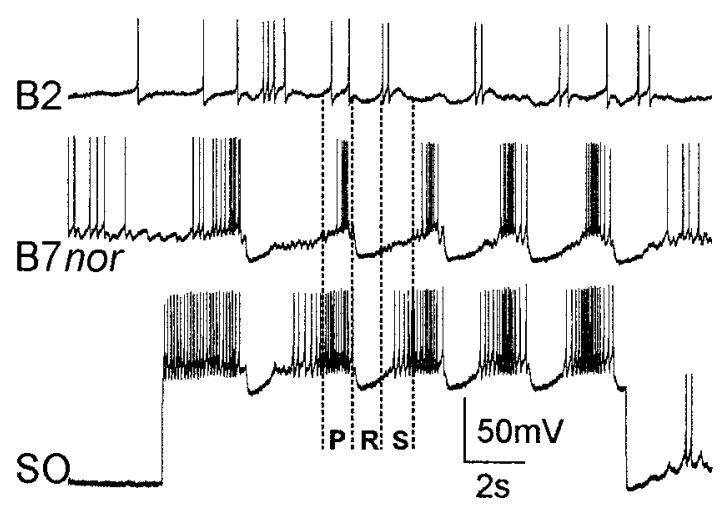

Figure 2. Transmission between $\mathrm{B} 2$ and B7nor and the activity of B7nor during fictive feeding. $A$, In physiological saline, brief bursts of $\mathrm{B} 2$ activity, but not single action potentials, typically elicit a slow depolarization in the follower neuron (upper) that can reach spiking threshold (lower). B, In HiDi saline, B2 action potentials produce constant latency, one-to-one unitary EPSPs in the B7nor neuron. The oscilloscope was triggered from the rising phase of the $\mathrm{B} 2$ action potentials, and five sweeps are superimposed. $C$, Simultaneous recordings from the B2, B7nor, and SO neurons are shown. The feeding CPG is activated by depolarization of the SO. Note that rhythmic activity in B7nor is entrained to the feeding CPG. A brief burst of spikes occurs during the protraction phase $(P)$; the neuron is inhibited during rasping $(R)$ and recovers in the swallowing phase $(S)$. In this example, the B2 neuron is only weakly active. Although single B2 action potentials occur at irregular intervals throughout the record, note that bursts of two to four B2 action potentials always occur at the end or just after the rasp phase. under these conditions and was enhanced sufficiently to reveal constant latency unitary synaptic events in B7nor, a monosynaptic excitatory connection is likely to exist between the two identified neurons. This conclusion is consistent with the overlapping fields of arborization for the two neurons (Fig. 1D) and is further strengthened by results obtained in cell culture (see below).

\section{B7nor participates in feeding behavior}

The B2 neuron is well established as an esophageal motoneuron (Benjamin and Rose, 1979; Benjamin et al., 1979; Perry et al., 1998), and its spiking activity is weakly entrained to the feeding CPG. Because the buccal ganglia are the primary location for the neurons of the feeding CPG and because the B7nor innervates the buccal mass feeding apparatus, it was of interest to consider whether this neuron also has a role in feeding. In isolated preparations of the CNS, the feeding CPG can be activated by stimulating an identified modulatory neuron in the buccal ganglion (see Fig. $1 B$ for cell body location) known as the slow oscillator or SO (Rose and Benjamin, 1981; Elliott and Benjamin, 1985a,b; Yeoman et al., 1993). This pattern is called "fictive" feeding and consists of three repeated phases of activity that underlie the three components of the feeding cycle in the intact animal, namely, protraction of the toothed radula and rasping of the radula against the substrate followed by swallowing. During a bout of SO-driven fictive feeding, B7nor activity is entrained to the feeding CPG, with spiking activity occurring during the protraction phase, inhibition occurring during rasping, and recovery from inhibition occurring during swallowing (Fig. 2C).

\section{Nitrergic pharmacology of the B2 to B7nor EPSP}

Various types of experiments were performed to determine whether NO mediates the depolarization evoked by B2 activity in B7nor.

First, we used two inhibitors of NOS, L-NNA and L-NAME. L-NNA ( $1 \mathrm{~mm})$ causes an almost complete [to $2 \pm 1 \%$ of control; $t$ test (control vs L-NNA), $p<0.001 ; n=3$ ] and irreversible block of the EPSP (Fig. $3 A, D$ ). The second NOS inhibitor L-NAME (1 $\mathrm{mM}$ ) also causes a significant block [51 $\pm 8 \%$; $t$ test (control vs L-NAME), $p=0.003 ; n=4]$ that in the time course of our experiments $(\sim 1 \mathrm{hr})$ is not reversed (Fig. $3 B, D)$. In contrast, the $\mathrm{D}$-isomer of NAME at the same concentration does not reduce the amplitude of the EPSP [94 $\pm 4 \%$; $t$ test (control vs D-NAME), $p=0.14 ; n=3$; Fig. $3 C, D]$.

Second, the NO scavenger PTIO was used to remove NO from the preparation. PTIO (0.25 mM) almost completely suppresses the EPSP, an effect that is readily reversed by washing. The EPSP was reduced to $1 \pm 1 \%$ [ $t$ test (PTIO vs control), $p<0.001 ; n=$ $3]$ of its pretreatment amplitude (Fig. $4 A, B$ ) within 7 min. Recovery after washout was slower, requiring $\sim 20 \mathrm{~min}$, but EPSP amplitude was almost completely restored ( $93 \pm 16 \% ; n=3)$.

Third, the known cholinergic antagonist hexamethonium chloride that has been shown to block excitatory cholinergic EPSPs in Lymnaea stagnalis effectively (for example, Yeoman et al., 1993) was used to test for a possible involvement of $\mathrm{ACh}$ in the slow depolarization of B7nor caused by B2 activity. We believed that this was necessary because it has recently been shown that B2 contains ACh as its main classical transmitter (Perry et al., 1998). However, bath application of hexamethonium chloride $(0.5 \mathrm{~mm})$ for prolonged periods had no effect on the amplitude of the slow depolarization ( $n=4$; data not shown). Experiments performed on isolated cultured B7nor neurons also show that the slow EPSP is not cholinergic (see below). 
A

B
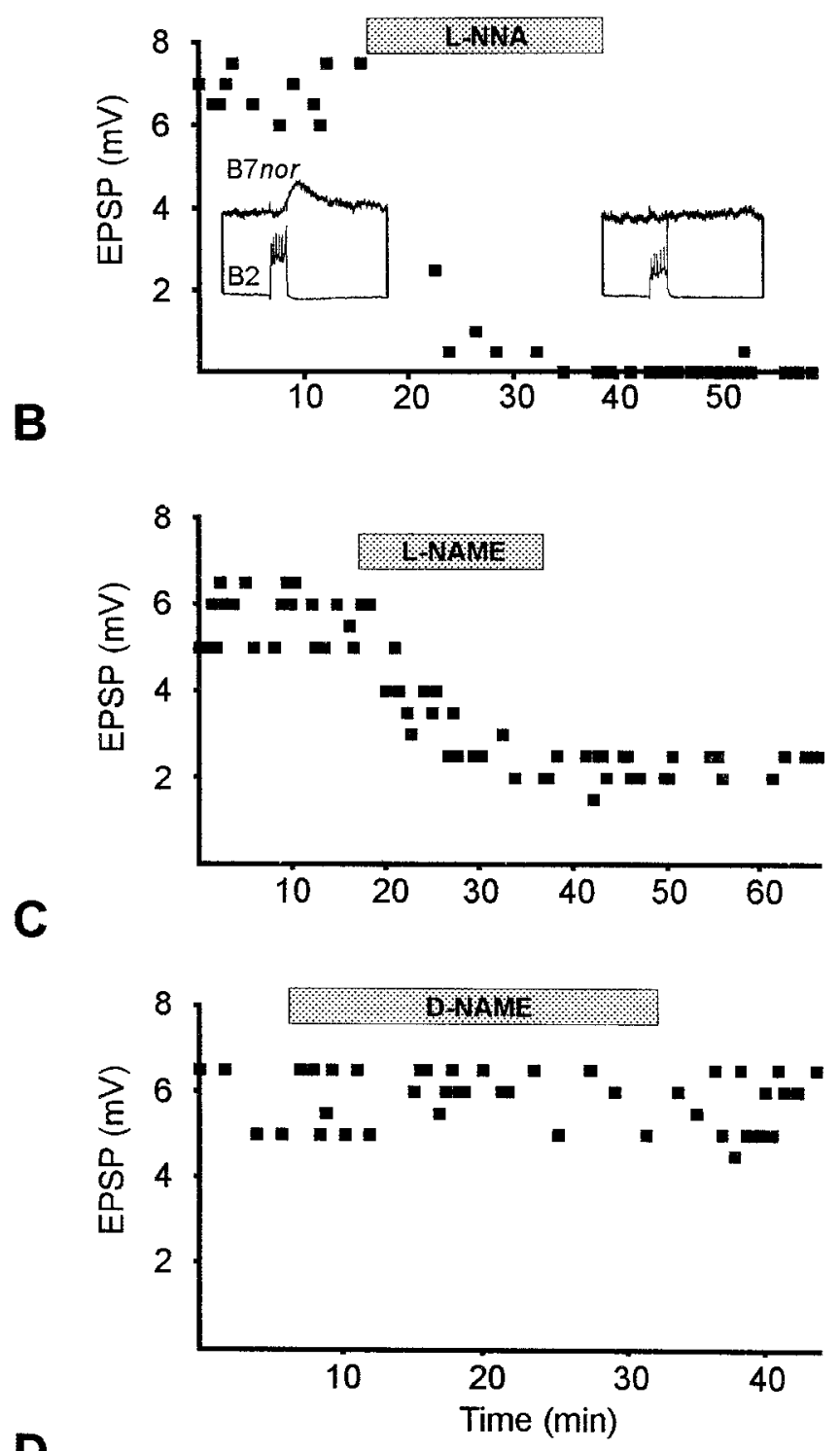

D

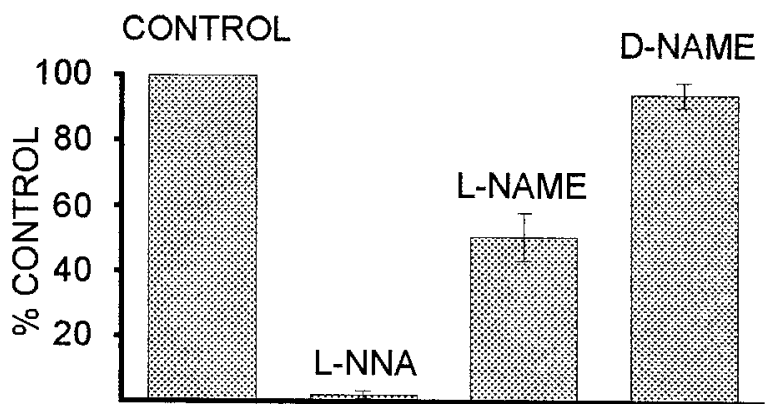

Figure 3. Effect of NOS inhibitors on transmission from B2 to B7nor in the intact CNS. $A$, Treatment with L-NNA ( $1 \mathrm{~mm}$; shaded box) causes a rapid decline in the amplitude of the EPSP that in the experiment illustrated is completely eliminated in $\sim 15 \mathrm{~min}$ and did not recover during the $1 \mathrm{hr}$ experiment. Sample intracellular recordings (insets) from the two neurons are shown before and at the end of the L-NNA application. The current injected into the presynaptic B2 neuron was the same in each test and produced approximately equivalent bursts of $\mathrm{B} 2$ action potentials. $B$, L-NAME (1 mM) treatment also reduces the amplitude of the B2-induced EPSP, but in comparison with L-NNA, the effect is slower and incom-
A

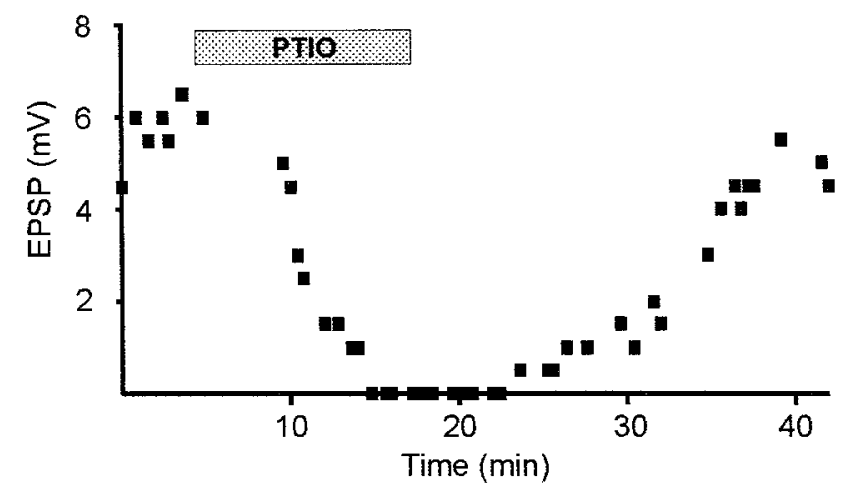

B

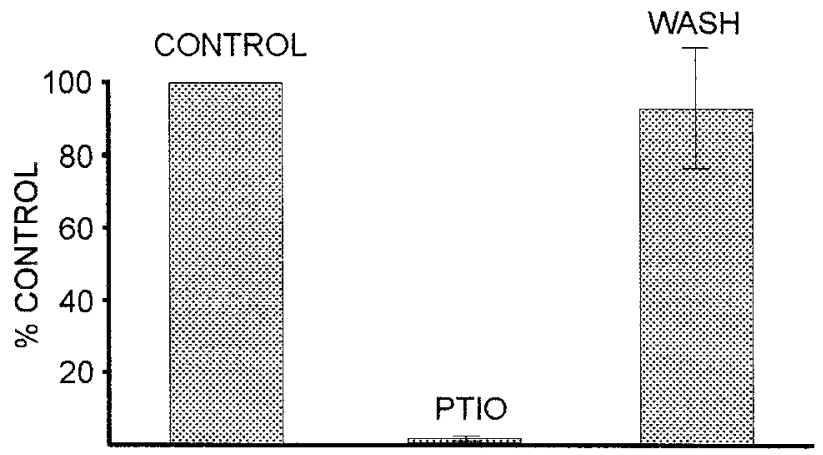

Figure 4. The NO scavenger PTIO produces a reversible suppression of the B2-induced EPSP in B7nor in the intact CNS. A, A sample treatment with PTIO $(0.25 \mathrm{~mm})$ in which PTIO causes a rapid decrease in the EPSP amplitude and completely abolishes the depolarization. The block is readily reversed after removal of PTIO from the bath. $B$, Summary of the PTIO experiments with average changes given as percentages \pm SEM. PTIO treatment blocks $99 \pm 1 \%(n=3)$ of the transmission, but the EPSP amplitude shows an almost complete recovery after washout of PTIO and returns to $93 \pm 16 \%(n=3)$ of the control value.

Fourth, we tested for a potential involvement of the two major peptides myomodulin A and SCP known to be present in B2 (Santama et al., 1994). Unfortunately, no specific inhibitors are available to interfere with myomodulin or SCP signaling. Therefore, these compounds were focally applied to B7nor neurons in the intact CNS by pressure ejection from a micropipette positioned above the cell body. Both myomodulin A (pipette concentration, $0.1 \mathrm{mM}$ ) and an equimolar mixture of the two forms of $\mathrm{SCP}, \mathrm{SCP}_{\mathrm{A}}$ and $\mathrm{SCP}_{\mathrm{B}}$ (both at pipette concentrations of $0.1 \mathrm{~mm}$ ),

$\leftarrow$

plete, declining in this example to approximately one-third of the control value in $25 \mathrm{~min}$. $C$, A $27 \mathrm{~min}$ exposure to D-NAME (1 mM) has no effect on EPSP amplitude in this experiment. Note that the data in $C$ is continuous with and precedes data taken from the same preparation shown in $B$. Thus the D-NAME treatment was followed by the superfusion of L-NAME. $D$, A summary of the effects of NOS inhibitors expressed as a percentage of control (pretreatment EPSP amplitude) \pm SEM is shown. On average, L-NNA $(1 \mathrm{mM})$ causes a decrease of the EPSP amplitude to $2 \pm 1 \%(n=3)$ of the control value. L-NAME $(1 \mathrm{mM})$ reduced the EPSP amplitude to $51 \pm 8 \%(n=4)$ of the control. D-NAME has no significant effect $(94 \pm 4 \% ; n=3)$. 
had no discernible effect on the membrane potential of B7nor (several trials; two preparations; data not shown).

\section{Microfocal application of NO and NO donors}

Further evidence of the involvement of NO in the B2-evoked EPSP was provided by the results of experiments in which NO was focally applied to B7nor neurons in the intact CNS. For this purpose, either a saturated solution of NO or one of two donor compounds, SNC and SNAP, was pressure ejected onto the cell bodies of B7nor neurons. In control experiments, saline, degassed SNC, and NAP were used to check for NO-unrelated effects of the carrier solutions or donor compounds.

The effects on the B7nor neuron of multiple pressure pulses delivered to a pipette containing NO-saturated saline is shown in Figure $5 A$ (upper trace). Brief single pulses of NO-saturated saline had no effect on the B7nor membrane potential, but a series of pulses at $10 \mathrm{~Hz}$ for $\sim 10 \mathrm{sec}$ caused a consistent slow depolarization (12 trials in $n=3$ preparations) that triggered a series of B7nor action potentials. In comparison, a similar series of saline pulses had no consistent effect on B7nor neurons (Fig. 5A, lower trace).

Focal application of the NO donor SNC to the B7nor cell body induced generally much stronger membrane depolarizations than did NO-saturated saline. A depolarizing response in excess of 25 $\mathrm{mV}$, associated with a burst of action potentials, can be elicited by a single pressure pulse of $20 \mathrm{msec}$ duration delivered to the pipette containing $100 \mathrm{~mm}$ SNC positioned over the B7nor cell body at a distance of $\sim 50 \mu \mathrm{m}$ (Fig. 5B, upper trace). Other neurons in the vicinity, including the B2 neuron, however, are unaffected. The depolarization can be attributed to the release of NO by SNC, because degassed SNC solutions failed to excite B7nor neurons in control experiments in the same preparations that showed depolarizing responses to fresh SNC solutions (Fig. $5 B$, lower trace).

In addition to SNC, a second NO donor, SNAP, was tested for its ability to elicit depolarizing responses in B7nor neurons. A typical response to the application of a single pressure pulse of SNAP (pipette concentration, $50 \mathrm{~mm}$ ) is shown in Figure $5 \mathrm{Ci}$. The response elicited by SNAP very closely resembles the slow depolarization of B7nor caused by B2 activity in the same preparation (Fig. 5Cii). The control compound NAP is unable to release NO and failed to elicit a response in any of these preparations (Fig. $5 \mathrm{Ci}$ ). These results further support the conclusion that the B2B7nor interaction can be mimicked with NO.

The observation that NO-saturated saline generally causes a weaker depolarization than that caused by the NO donors SNAP and particularly SNC is possibly because the NO concentration in the pipette is significantly less than saturated because of local diffusion and oxidative effects in the vicinity of the tip. These effects are likely to be more significant for aqueous solutions of NO than for donor solutions, because for the latter the NO concentration is restored by the donor.

\section{Coculture of the identified B2 and B7nor neurons}

After the electrophysiological identification of B7nor neurons and the identification of B2 neurons by visual inspection, both were isolated from the CNS together with a short segment of their primary neurites and cocultured. Within the first $6 \mathrm{hr}$ in culture, the neurons usually retracted their neurites before extending new processes. In some cases a section of the original primary neurite was retained, and in these cases the initial outgrowth of new processes occurred predominantly at the tip. In other cases, the
A
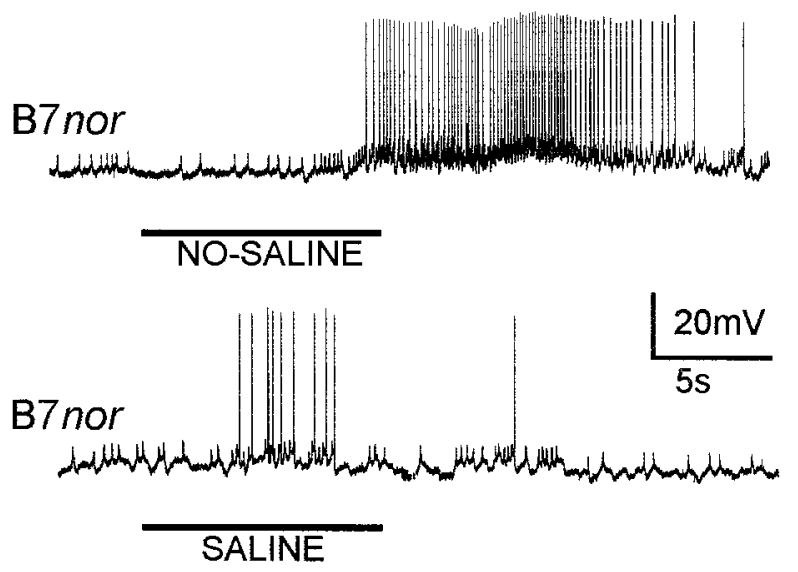

B
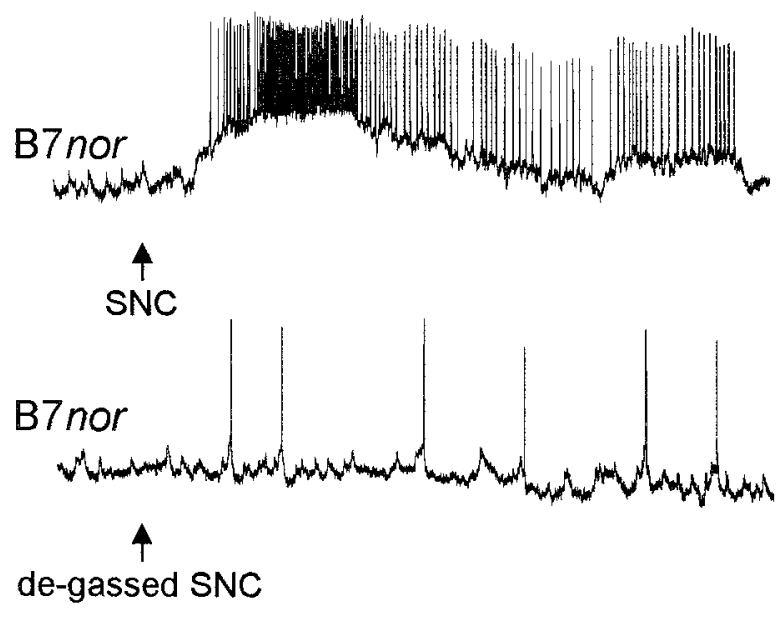

$\mathbf{C i}$

Cii
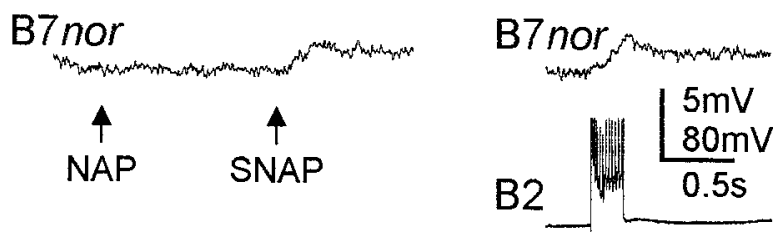

Figure 5. Focal application of NO-containing solutions to the B7nor neuron in the intact CNS. $A$, Effect of NO-saturated saline (pipette concentration, $<2 \mathrm{~mm}$; see Materials and Methods) ejected by a train of pressure pulses of $200 \mathrm{msec}$ duration at $1 \mathrm{~Hz}$ for the period indicated by the horizontal line (upper trace). The pipette tip was placed $\sim 50 \mu \mathrm{m}$ from the cell body of the B7nor neuron. The NO-saturated saline caused a depolarization accompanied by a prolonged burst of B7nor action potentials. The lower trace shows that the ejection of saline without NO has no effect. $B$, Effect of a single pulse of fresh SNC at $100 \mathrm{~mm}$ (upper trace) or of a degassed and nitrogen-purged solution of SNC (lower trace). Fresh SNC caused a strong depolarization of B7nor that triggered the generation of a burst of activity. In contrast, degassed SNC had no consistent effect. $C i$, Effect of single pressure pulses of SNAP and NAP. SNAP (50 $\mathrm{mM} ; 40 \mathrm{msec})$ but not NAP $(50 \mathrm{~mm} ; 40 \mathrm{msec})$ caused a consistent depolarization in B7nor. Cii, Effect of a brief burst of B2 action potentials. In the same preparation shown in $\mathrm{C} i$, this burst causes the expected depolarizing response in B7nor that is directly comparable with the effect of SNAP (also see Fig. 11 for the effect of SNAP on isolated cultured neurons). 


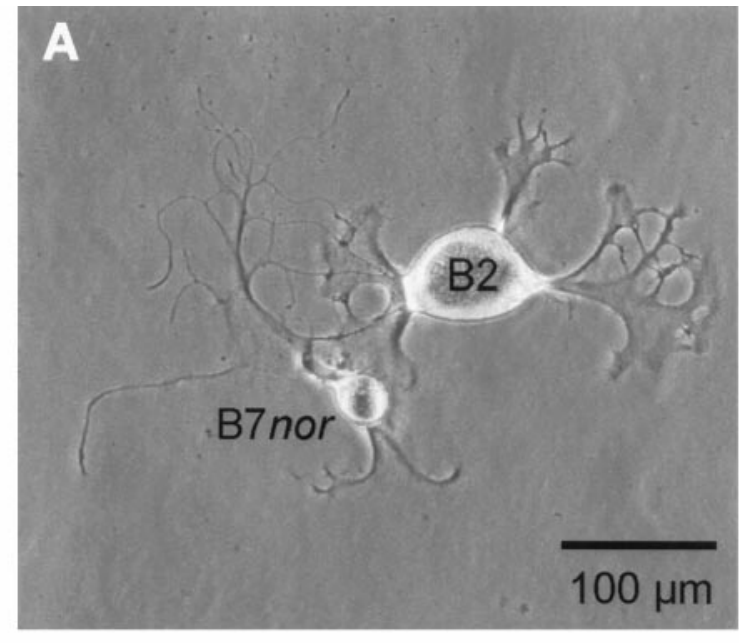

B

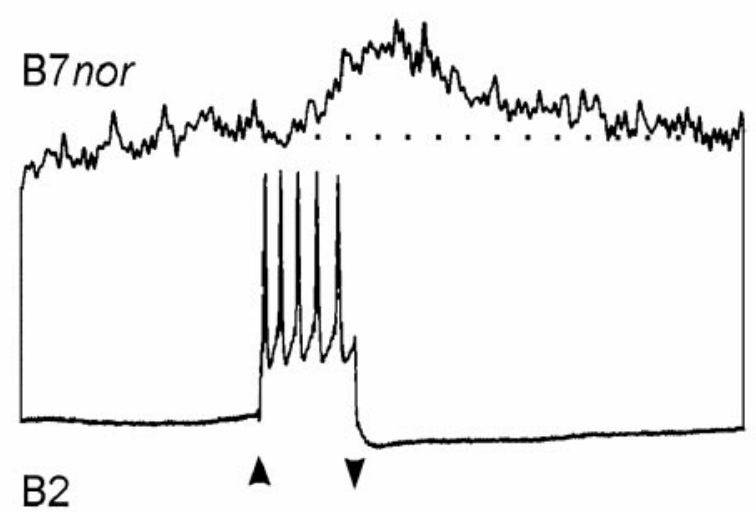

C

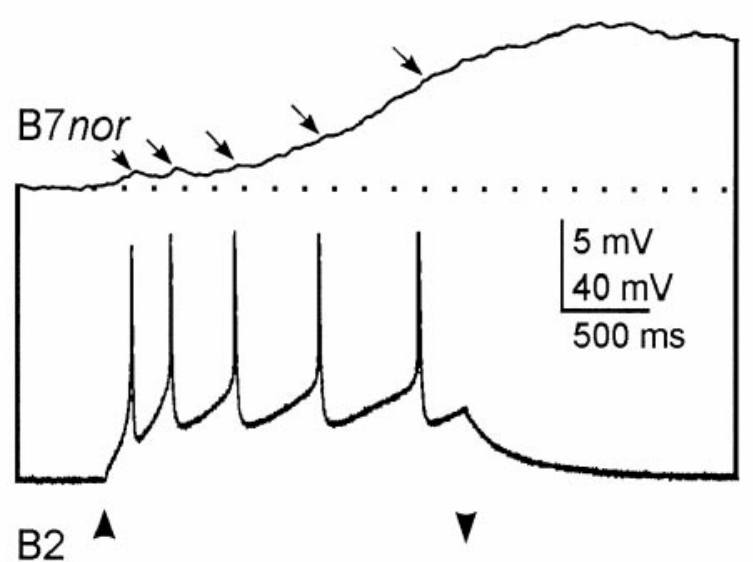

Figure 6. Reconstitution of the B2-B7nor interaction in cell culture. $A$, Photomicrograph of identified B2 and B7nor neurons after $1 \mathrm{~d}$ in cell culture is shown. Both neurons have regenerated extensive new processes and have already established contact after their isolation from the buccal ganglion. Note the relatively large growth cones at the tips of the processes. $B$, In the intact CNS before their isolation, a burst of five action potentials in the $\mathrm{B} 2$ neuron $(\sim 10 \mathrm{~Hz})$ triggered by current injection caused a slow depolarization of the B7nor neuron that began $\sim 200 \mathrm{msec}$ after the start of $\mathrm{B} 2$ activity and peaked at $\sim 0.7 \mathrm{sec}$. $C$, Two days neurons extended multipolar processes from the cell body. Overall, $\sim 70 \%(n=76)$ of isolated B7nor neurons extended processes in culture. A comparable proportion of isolated B2 neurons ( $72 \%$; $n=95$ ) grew processes in culture, indicating that the injection of fast green into B7nor cells did not affect their regenerative properties. After the initial outgrowth that occurred usually within the first $12 \mathrm{hr}$ after isolation, growth and elaboration of processes normally continued for a period of 3-4 d. Neurons could be kept in culture for up to $10 \mathrm{~d}$ before they showed marked signs of deterioration. When pairs of B2 and B7nor neurons were cocultured in close proximity $(<500 \mu \mathrm{m})$, they readily grew overlapping processes, making physical contact within the first $2 \mathrm{~d}$ of culture (Fig. $6 \mathrm{~A}$ ).

\section{Reconstitution of B2 to B7nor neurotransmission in cell culture}

Using the coculture approach, we have demonstrated that an interaction occurs between B2 and B7nor that has the characteristic features of the synapse in the intact CNS. Stimulation of B2 neurons results in slow depolarizations of B7nor neurons in 79\% of all cases in which simultaneous recordings were made from pairs of the cocultured neurons that had extended overlapping processes ( 42 out of 53 cell pairs tested). A direct comparison of the interaction between individual $\mathrm{B} 2$ and $\mathrm{B} 7$ nor neurons before and after their isolation from the brain shows that the synapse is somewhat stronger and slower in cell culture (Fig. 6B,C). Thus the connection is rarely strong enough to reveal unitary synaptic events in the intact brain, whereas single action potentials typically produce unitary slow depolarizing potentials in cell culture. Despite these significant differences, the response in cell culture is qualitatively remarkably similar.

Single action potentials in the B2 neuron typically cause a slow depolarization of $\sim 2 \mathrm{mV}$ at membrane potentials of approximately $-70 \mathrm{mV}$ (Fig. 7 $\mathrm{Ai}$ ). Brief bursts of action potentials at 1-3 $\mathrm{Hz}$ result in a summating response after the second action potential (Fig. 7Aii-Aiv) that leads to a linear increase in EPSP amplitude (Fig. $7 B$ ). Electrical coupling between the cocultured neurons can be demonstrated by injecting negative current pulses into either neuron (see Materials and Methods) and occurs in $51 \%$ (27 out of 53 cell pairs tested) of the pairings. Thus in approximately one-half of our recordings, this coupling resulted in the appearance of fast electrical EPSPs in the B7nor neuron that are one-to-one with action potentials in $\mathrm{B} 2$ and precede the slower response (for example, see Fig. 7Ai-Aiv, also see Fig. 6C).

\section{Anomalous cholinergic transmission in cultured cells}

In addition to the slow excitation and the electrical coupling, a novel inhibitory interaction between cultured B2 and B7nor neurons was observed in 8 out of 53 cell pairs tested. We refer to this

after the isolation of the same two neurons, an excitatory connection was restored in cell culture. The injection of a depolarizing current into the isolated B2 neuron caused a burst of five action potentials but at a lower frequency $(\sim 2.5 \mathrm{~Hz})$. The $\mathrm{B} 2$ activity produced a slow depolarization in the B7nor neuron that peaked $\sim 2.5 \mathrm{sec}$ after the start of B2 activity. Weak, brief depolarizing events (indicated by small arrows) are caused by electrotonic coupling between B2 and B7nor and are seen in approximately one-half of the cocultured preparations examined (see also Figs. 7, $9-11)$. The start and the end of the current injection are indicated by up and down arrowheads, respectively, in $B$ and $C$. The records in the intact CNS and in cell culture are shown at the same scale. The vertical bar is equivalent to 5 and $40 \mathrm{mV}$ for the $\mathrm{B} 7$ nor and $\mathrm{B} 2$ recordings, respectively. 

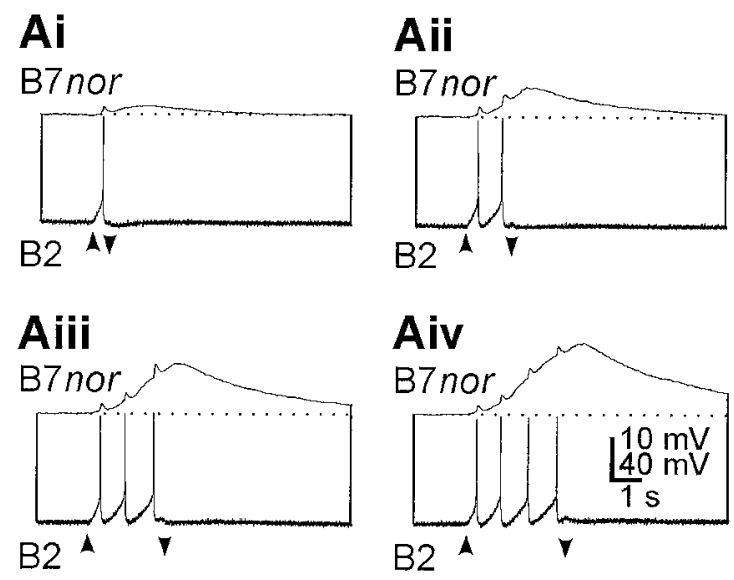

B

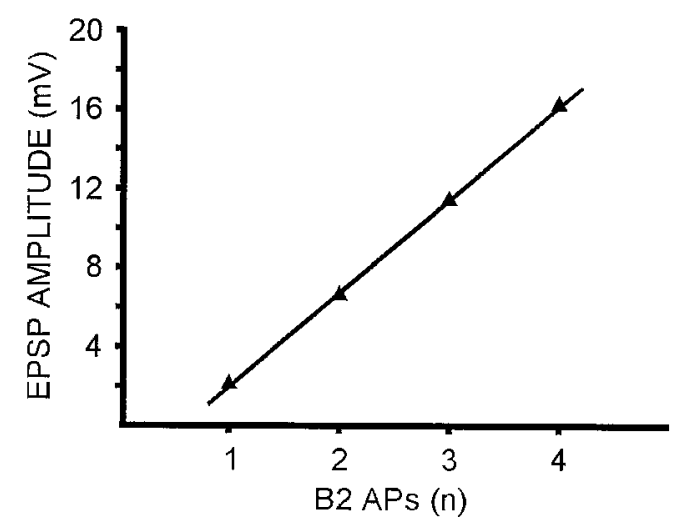

Figure 7. Relationship between the number of $\mathrm{B} 2$ action potentials and the size of the B7nor response in cell culture. Ai-Aiv, Incremental length, constant amplitude current pulses were injected into a B2 neuron to trigger one, two, three, or four action potentials. The start and the end of each current pulse are indicated by the up and down arrowheads, respectively. B2 activation caused a slow depolarization in B7nor that summates with increasing numbers of B2 action potentials. In addition to the slow depolarization, each individual B2 action potential also caused a brief depolarization of the B7nor membrane potential because of electrotonic coupling. All records are shown at the same scale. $B$, Plotting the amplitude of the B7nor EPSP against the number of B2 action potentials showed a linear increase in EPSP amplitude.

as anomalous because this was never seen in the intact CNS. In these exceptional cases, B2 action potentials were followed by a biphasic response in B7nor neurons consisting of a fast unitary IPSP that preceded the slower EPSP (Fig. $8 A$ ). Interestingly, this biphasic response was observed in all cases in which B2 and B7nor had established soma-soma contact in cell culture $(n=4)$ and in four cases in which B2 processes appeared to make direct somatic contacts with B7nor. However, fast IPSPs were never seen when cocultured B2 and B7nor neurons had established only contacts between processes.

The fast unitary IPSPs but not the slower EPSPs were completely abolished by perfusing the culture dish with d-TC $(0.1$ mM; Fig. $8 B$ ), an acetylcholine antagonist that has been shown to block inhibitory and excitatory cholinergic interactions in Lymnaea stagnalis (e.g., Yeoman et al., 1993). The fast unitary IPSPs were restored when the d-TC was exchanged for L-NAME (1 mM) that, as expected, blocked the slow EPSP (Fig. 8C). The susceptibility of the slow interaction between cultured B2 and B7nor neurons to block by L-NAME will be described in more detail below. The IPSP was also mimicked by the focal application of acetylcholine ( $n=4$; data not shown).

These results show that $\mathrm{B} 2$ and $\mathrm{B} 7$ nor neurons are able to form inhibitory cholinergic synapses in cell culture, but it is clear that acetylcholine is not the transmitter of the slow EPSP evoked in B7nor neurons by B2 activity.

\section{Pharmacological interference with NO signaling between cocultured neurons}

Our experiments in the intact CNS indicated that NO mediates the anterograde excitatory interaction between B2 and B7nor neurons. To support this hypothesis further, we have performed a series of experiments on cocultured B2 and B7nor neurons in which the effect of the NOS inhibitor L-NAME, its inactive optical isomer D-NAME, and the NO scavenger PTIO are examined.

Superfusing the culture with D-NAME $(0.1 \mathrm{~mm})$ while recording the response of $\mathrm{B} 7$ nor neurons to $\mathrm{B} 2$ stimulation did not reduce the EPSP amplitude. On the contrary, in two out of three experiments, an increase in the PSP amplitude, in one case by $40 \%$, was observed after the application of D-NAME. In the experimental results shown, the EPSP amplitude remained unaltered during the application of D-NAME (Fig. 9A,B). On average, however, the EPSP amplitude was increased by $\sim 20 \%(t$ test control vs D-NAME, $p=0.14$, not significant; $n=3$ ) by D-NAME. This effect was reversed by the application of L-NAME $(0.1 \mathrm{mM})$ that significantly reduced the size of the PSP by an average of $45 \%$ ( $t$ test control vs L-NAME, $p=0.003 ; n=$ 3 ) compared with the control value after 45 min of application (Fig. 9Aiii, C). The effect of L-NAME was partially reversible, and the EPSP amplitude could return to up to $90 \%$ (Fig. 9Aiv) of the control values after a prolonged period of washing the preparation in normal saline. On average, the EPSP amplitude returned to $68 \%$ of the control value $(n=3$; Fig. $9 C)$. Using L-NAME at a higher concentration $(1 \mathrm{~mm})$ led to a more rapid and complete block of the synaptic interaction, reducing the amplitude of the PSP by $\sim 60 \%$ within $20 \mathrm{~min}(n=2)$. The reversal of this effect is however also considerably slower and less complete than the reversal of the inhibition by L-NAME at the lower concentration.

The results of the experiments with NOS inhibitors provide evidence that $\mathrm{NO}$ is involved in the signaling between cultured B2 and B7nor neurons. This evidence is further supported by the observation that the slow component of the B2-B7nor interaction can be abolished almost completely by bath application of the potent NO scavenger PTIO (0.25 mm; Fig. $10 A i-A i i, B)$. The PTIO-resistant fast interaction was caused by electrotonic coupling between the two cultured neurons. PTIO reduced the amplitude of the slow EPSP component to $\sim 8 \%$ ( $t$ test control vs PTIO, $p<0.001$; $n=3$; Fig. $10 B, C)$ of the control value within $3 \mathrm{~min}$ (Fig. 10B). The effect was readily reversed after washout of PTIO from the bath (Fig. 10Aiii, B), the B7nor EPSP amplitude returning to an average of $62 \%$ of the control values $(n=3$; Fig. 10C).

\section{B2 stimulation versus NO donor application in cultured B7nor neurons}

Further evidence of the mediation of the slow depolarization from B2 to B7nor neurons was obtained by focal application of the NO donor SNAP onto the cell bodies of isolated B7nor neurons. The application of a pulse of SNAP (pipette concentration, $1 \mathrm{~mm}$ ) caused a consistent depolarization of the B7nor 
A

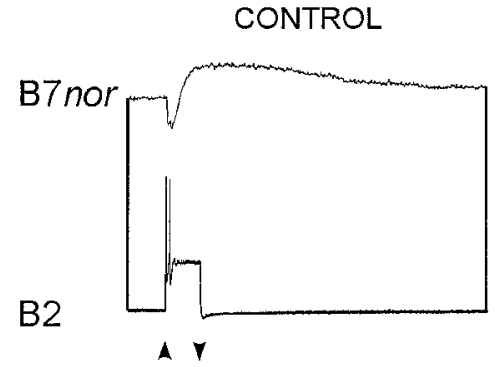

B

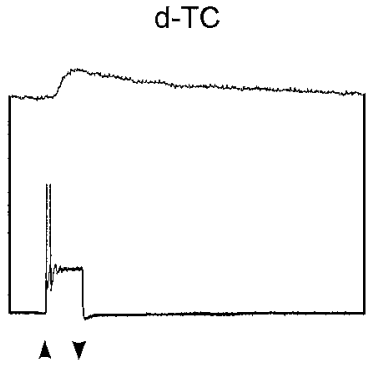

C

\section{L-NAME}

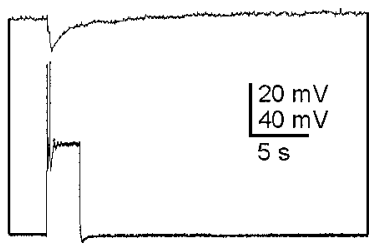

A

Figure 8. Anomalous cholinergic transmission from $\mathrm{B} 2$ to B7nor neurons in culture. A, Biphasic response of a B7nor neuron to $\mathrm{B} 2$ activity observed in a pair of neurons that had established soma-soma contact in cell culture is shown. Each of the two B2 action potentials that were triggered by the injection of a constant positive current pulse elicited a fast unitary IPSP in B7nor followed by a slower, delayed depolarization. $B$, Perfusing the bath with d-TC $(0.1 \mathrm{mM})$ completely abolished the fast IPSPs but had no significant effect on the amplitude of the slow depolarization. $C$, The fast IPSP was restored when the d-TC was exchanged for L-NAME $(1 \mathrm{mM})$, which blocked the slow depolarization. The start and end of the current pulses that triggered B2 activity are indicated by up and down arrowheads, respectively.

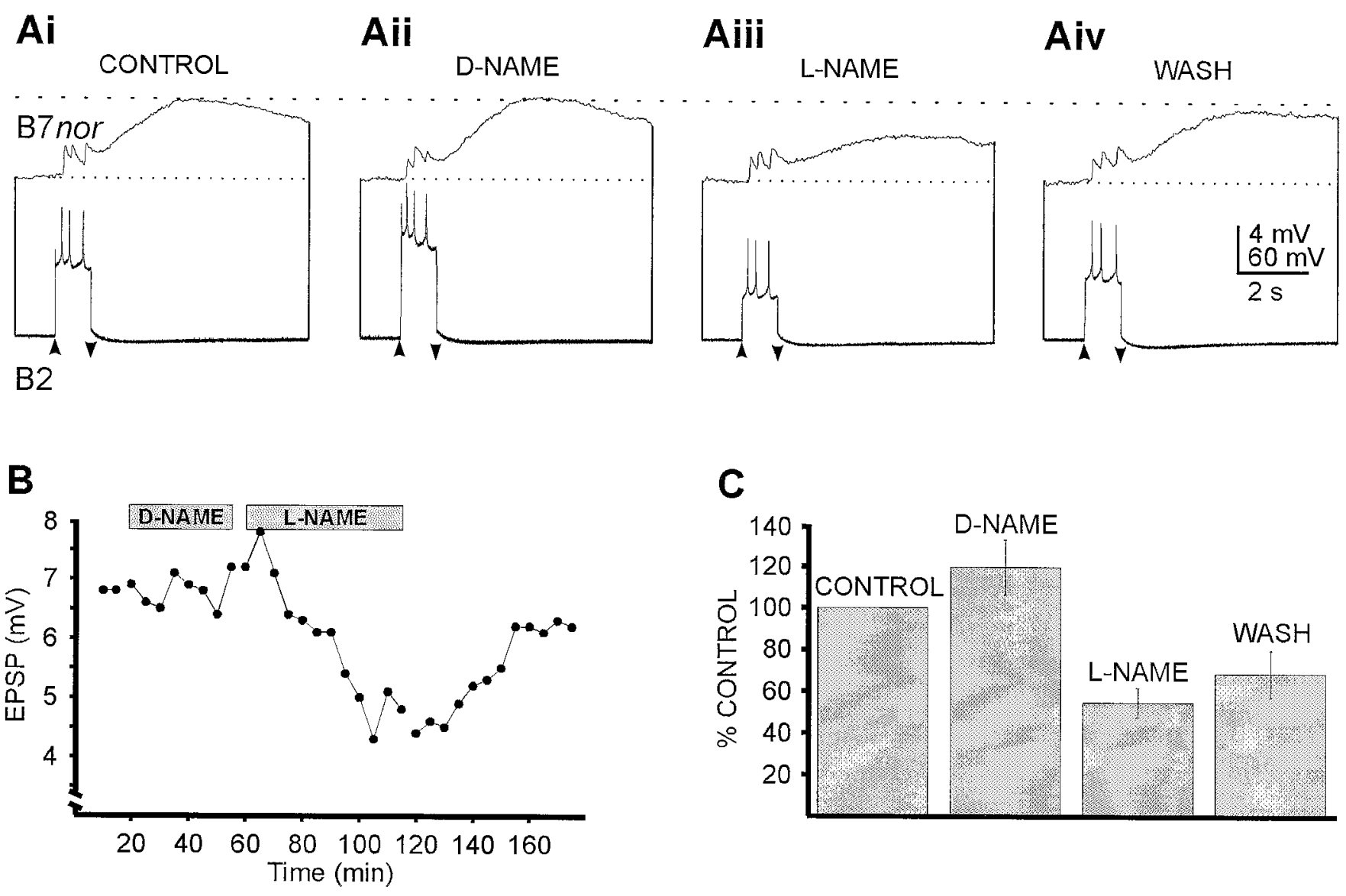

Figure 9. Effect of D- and L-NAME on the interaction between B2 and B7nor neurons in culture. Ai-Aiv, The injection of a constant current 1 sec pulse into a B2 neuron triggered a reproducible burst of three presynaptic action potentials. Electrotonic coupling between B2 and B7nor produced the initial fast one-to-one depolarizations of the B7nor membrane potential that were followed by a slowly developing depolarization. Aii, The amplitude of the slow EPSP was not effected by the application of D-NAME $(0.1 \mathrm{~mm})$ for $35 \mathrm{~min}$. Aiii, The application of L-NAME $(0.1 \mathrm{~mm})$ for $45 \mathrm{~min}$ reduced the slow EPSP amplitude from 6.9 to $4.3 \mathrm{mV}$. Aiv, After a $40 \mathrm{~min}$ washout of L-NAME, the EPSP amplitude had returned to $6.2 \mathrm{mV}$. All records are shown at the same scale. Up and down arrowheads indicate the start and end of the current pulse. B, Plot of the amplitude of B7nor EPSPs against time for the experiment shown in $A i-A i v$ illustrates the time course of the effects of D- and L-NAME applications. Each data point represents the B7nor response to a single burst of three B2 action potentials. The individual bursts were triggered at 5 min intervals. The period of D- and L-NAME application is indicated by a shaded box. C, Summary of three experiments showing the average changes in B7nor amplitude caused by applications of D- and L-NAME (both at $0.1 \mathrm{mM}$ ) is shown. The average changes are given as percentages ( \pm SEM) of the control response measured before the application of any drug. 

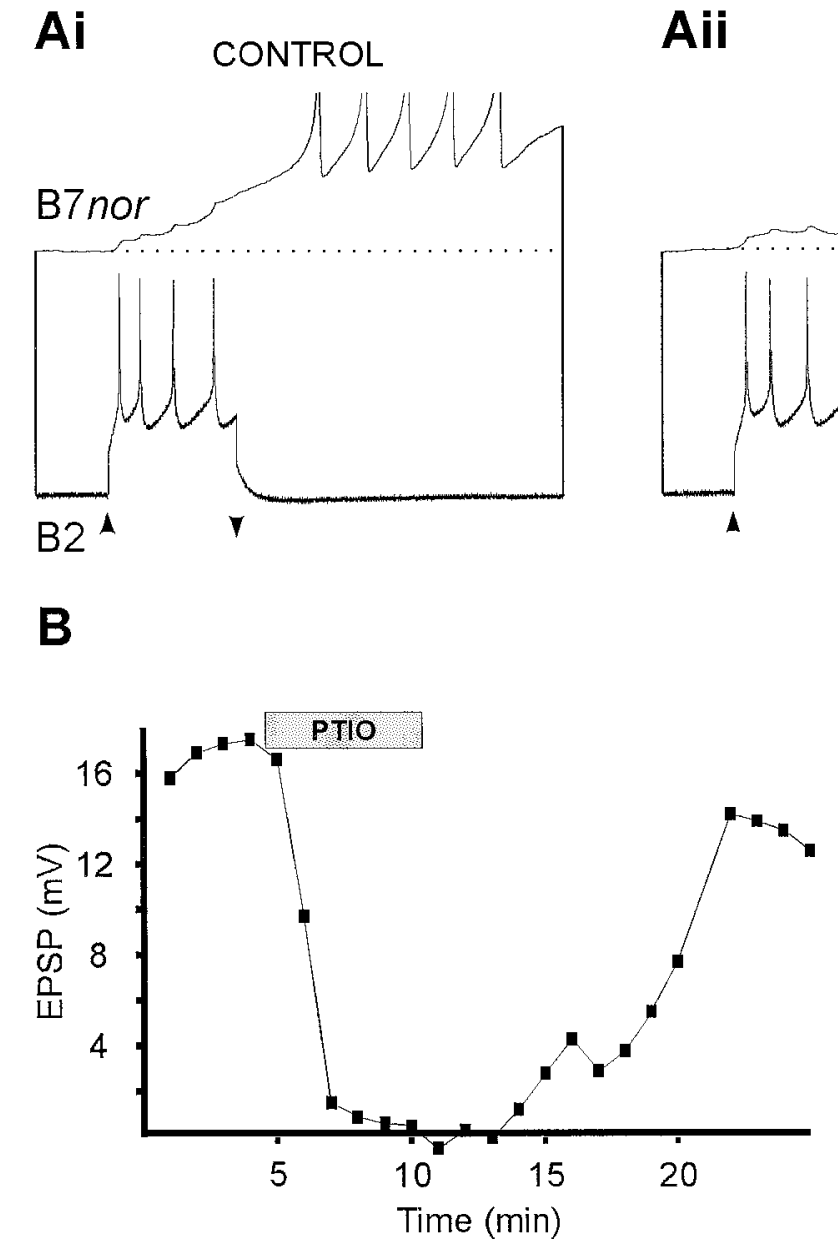

Aii

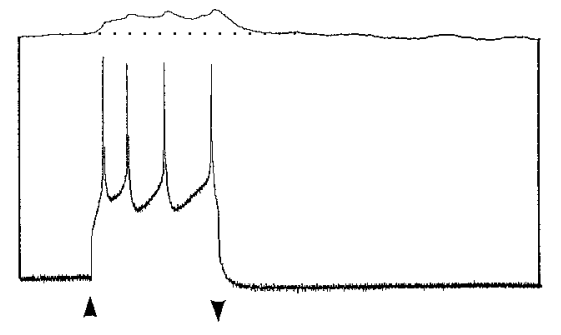

PTIO

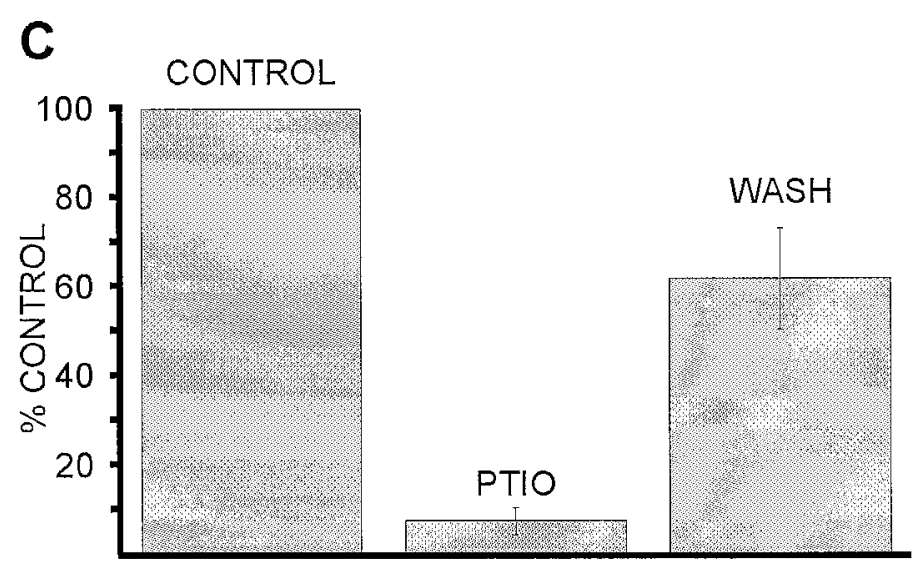

Figure 10. Effect of PTIO on the interaction between $\mathrm{B} 2$ and B7nor neurons in culture. Ai-Aiii, The injection of a constant current 2 sec pulse into a B2 neuron triggered a reproducible burst of four action potentials. Electrotonic coupling between B2 and B7nor produced fast one-to-one depolarizations of the B7nor neuron that are followed by a slowly developing depolarization that triggered a burst of action potentials in B7nor. Aii, The slow EPSP was abolished by the application of PTIO $(0.25 \mathrm{~mm})$ for $5 \mathrm{~min}$. Aiii, After a 13 min washout of PTIO, the amplitude of the slow EPSP had recovered sufficiently to trigger another burst of action potentials in B7nor. All records are shown at the same scale. Up and down arrowheads indicate the start and finish of the current pulse. $B$, Plot of the amplitude of B7nor EPSPs against time for the experiment shown in $A$ is presented. Note the rapid onset of the PTIO block and its ready reversal. Each data point represents the B7nor response to a single burst of four action potentials in B2. The individual bursts were triggered at $1 \mathrm{~min}$ intervals. The period of PTIO application is indicated by the shaded box. $C$, Summary of three experiments showing the average changes in B7nor amplitude caused by the applications of PTIO $(0.25 \mathrm{mM})$ is presented. The average changes are given as percentages $( \pm$ SEM $)$ of the control response measured before the application of PTIO.

membrane potential that was observed in all four neurons tested. The depolarization could trigger a burst of action potentials (Fig. $11 A$ ) and resembled the excitation caused by B2 activity (Fig. $11 C$ ). In contrast, the related control compound NAP, which cannot release NO, had no effect on any of the neurons tested (Fig. 11B).

The response of B7nor neurons both to SNAP and to stimulation of the B2 neuron was voltage-dependent. Hyperpolarization of the membrane potential caused a decrease in the amplitude of depolarization elicited by SNAP (Fig. 11D). A similar decrease in amplitude of the B2-evoked slow depolarization at reconstituted B2-B7nor synapses was also observed when the membrane potential of the B7nor neurons was hyperpolarized (Fig. 11E). In addition to this, both application of SNAP and stimulation of B2 cause a similar increase in the input resistance of B7nor neurons (from 15-30\%; data not shown). The input resistance of B7nor neurons, however, decreases when the membrane potential is hyperpolarized, and it is therefore possible that the change caused by SNAP or B2 activity is a secondary consequence of the depolarization elicited by them. We note, however, that in Aplysia NO has been reported to cause a decreased conductance EPSP and it has been suggested that this is caused by a decrease in potassium conductance (Jacklet, 1995).

The NO scavenger PTIO blocks the slow depolarization in B7nor neurons caused by B2 activity both in the intact CNS and in cell culture (Figs. 4, 10). PTIO was also able to suppress the effect of focal SNAP application on isolated B7nor neurons $(n=$ 2 ; data not shown). This represents another similarity between the depolarizations caused by SNAP and B2 activity and further supports our conclusion that NO is the excitatory transmitter between the $\mathrm{B} 2$ and $\mathrm{B} 7$ nor neurons.

\section{Spatial aspects of NO signaling in cell culture}

Recordings from B2 and B7nor neurons that had not yet established physical contact in cell culture showed a similar slow depolarization of the B7nor neuron after B2 stimulation (Fig. $12 A$ ). Considerably stronger and longer stimulation to the $\mathrm{B} 2$ neuron was, however, required to elicit consistent responses. In 

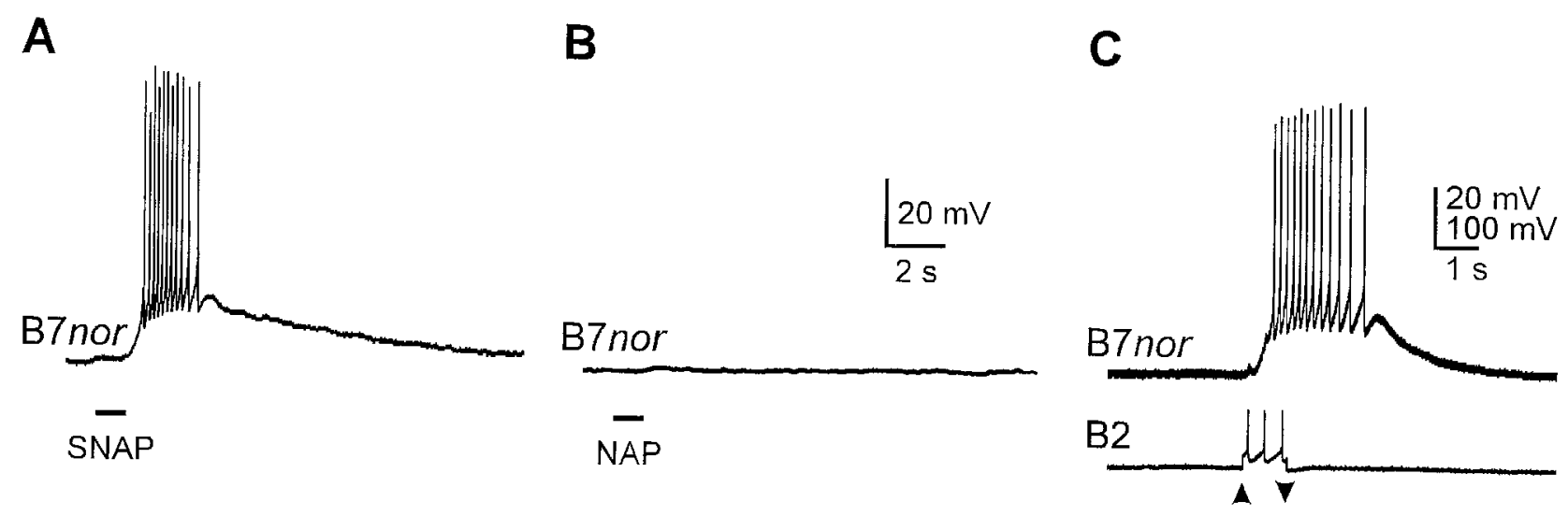

D

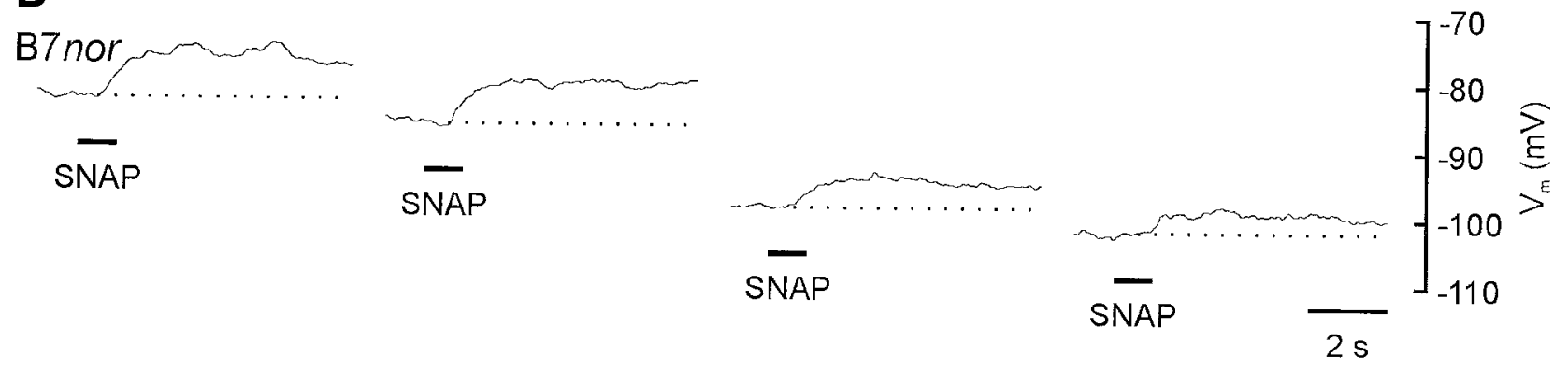

$\mathbf{E}$
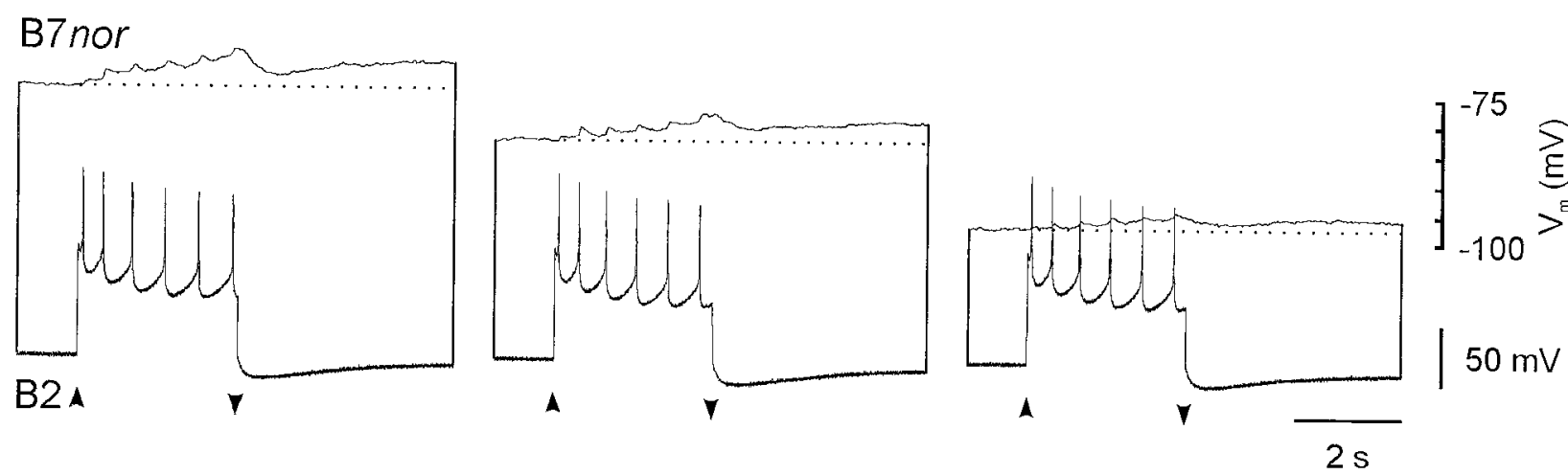

$50 \mathrm{mV}$

Figure 11. Effect of SNAP and NAP on an isolated B7nor neuron and comparison with reconstituted B2-B7nor synaptic interaction. A, Focal application (horizontal line) of a pulse of SNAP $(1 \mathrm{mM} ; 1 \mathrm{sec})$ caused a depolarization of the B7nor membrane potential that triggered the generation of a burst of action potentials. $B$, The application of a similar pulse of NAP $(1 \mathrm{mM} ; 1 \mathrm{sec})$ had no effect on the membrane potential of the same B7nor. $C$, Activity in a B2 neuron that had grown overlapping processes with a B7nor neuron in cell culture depolarized the B7nor neuron and triggered a burst of action potentials. The time course of the depolarization is comparable with the effect of SNAP shown in $A$. B2 activity was triggered by the injection of a depolarizing current (start and end indicated by up and down arrowheads, respectively). $D$, Hyperpolarization of the membrane potential of an isolated B7nor caused a reduction in the response to the application of SNAP (1 mM; $1 \mathrm{sec})$. A linear relationship existed between the amplitude of the SNAP response and the membrane potential (data not shown). E, Recordings from a pair of B2 and B7nor neurons that had established physical contact in cell culture are shown. A series of B2 action potentials was triggered by the injection of a constant depolarizing current pulse, the start and end of which are indicated by the up and down arrowheads, respectively. Each B2 action potential was followed by a fast PSP in B7nor that was caused by electrotonic coupling between the two neurons. (Electrotonic coupling between the neurons was tested for by injection of a series of constant negative current pulses in either of the two neurons; data not shown.) The fast electrotonic PSPs were followed by a slow depolarization that decreased in amplitude when the membrane potential was hyperpolarized.

two experiments, B2 neurons that had not grown any processes could be detached from the substrate and moved into different positions relative to the B7nor neuron, while both neurons were still impaled by microelectrodes. The EPSP amplitude increased when the distance between the B2 and B7nor neurons was re- duced (Fig. 12B,C). However, even when the cells were brought into direct contact, the interaction was weaker than the interaction between pairs of neurons that had arborized and formed overlapping fields, and the B2 neuron had to be stimulated strongly to achieve EPSP amplitudes of $>5 \mathrm{mV}$. This result, 

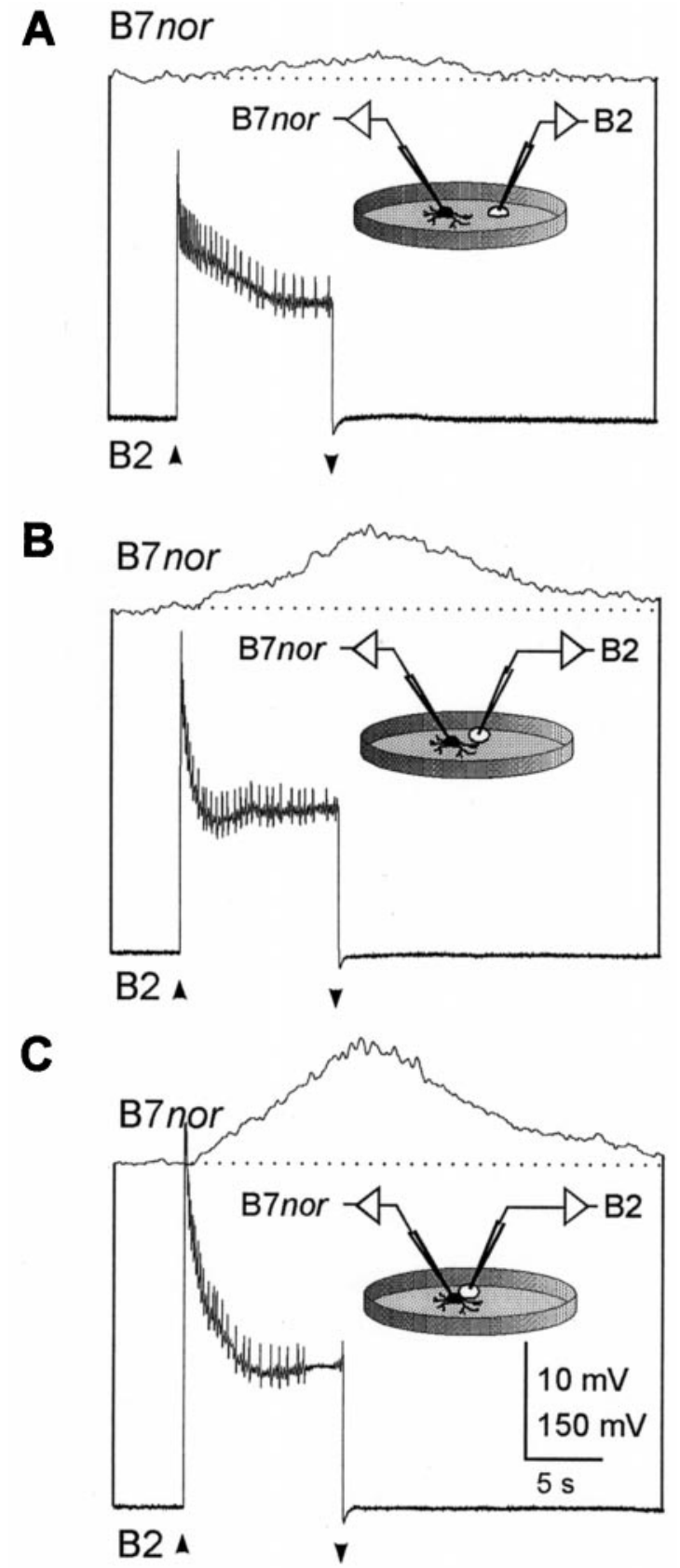

Figure 12. Nonsynaptic interaction between B2 and B7nor neurons in culture. $A-C$, Strong bursts of action potentials were triggered in a B2 neuron by injecting a constant, strong $10 \mathrm{sec}$ current pulse. All records are shown at the same scale. Up and down arrowheads indicate the start and finish of the current pulse. The inserts show a schematic representation of the recording configuration (not drawn to scale). $A$, At a distance of $\sim 40$ $\mu \mathrm{m}$ between the B2 and B7nor soma, the burst of B2 action potentials caused a weak depolarization with a peak amplitude of $2.2 \mathrm{mV}$. B, Manipulating the same B2 neuron with the recording electrode into a position $\sim 10 \mu \mathrm{m}$ from the B7nor soma resulted in an increase of the depolarization, which was caused by the B2 burst, to $7.2 \mathrm{mV}$. C, Approaching the $\mathrm{B} 2$ neuron further, so that it was in direct contact with the B7nor soma, increased the EPSP amplitude to $10.5 \mathrm{mV}$.

however, demonstrates that physical contact between the two neurons is not necessary and that NO released by B2 neurons can diffuse at least $40 \mu \mathrm{m}$ at physiologically effective concentrations. It also shows that naturally formed contacts considerably enhance the strength of the interaction.

\section{DISCUSSION}

Although NO is now regarded as a neuronal signaling molecule, it has proven difficult to define precisely its role in neurotransmission in the mammalian CNS. Difficulties arise in part from some unusual aspects of signaling by NO that cannot be accommodated by the classical model of the chemical synapse. For example, NO release does not require presynaptic specializations such as vesicles, and NO diffusion need not be confined to the extracellular space. Unconventional roles for NO as a long-range messenger and as a retrograde messenger have therefore been proposed (Hölscher, 1997). There seems little doubt that, when the NO signaling is more precisely understood, classical models of synaptic transmission and simplistic connectionist models of the brain will be challenged.

Although transmission by NO is unconventional, it is important to discuss the extent to which the generally accepted set of criteria that should be met to establish that a substance is a transmitter at a specific synapse can be fulfilled in our system. First, we and others (Moroz et al., 1994a,b) show that the presynaptic neuron expresses the enzyme required for the synthesis of NO. However, because NO cannot be stored in membrane-bound compartments, the related requirement to show presynaptic storage is inapplicable. Second, we show that the postsynaptic effect of B2 on B7nor can be blocked pharmacologically by inhibitors of NOS and by scavenging of released NO. Third, the effect of B2 on B7nor can be mimicked by $\mathrm{NO}$ and NO donor compounds. Thus both stimulation of B2 and NO application cause depolarizing responses, both effects are blocked by the NO scavenger PTIO, and both show a similar voltage dependence and are associated with an increase in input resistance. Fourth, although we have not identified the receptor in B7nor, we do report on the nonsensitivity to NO of other neurons, indicating a specialization in B7nor for selective NO receptivity. Finally, although it is among the classical criteria, we have not shown that there is a mechanism for specific inactivation of the putative transmitter. However, because NO is unstable and reactive, high-affinity uptake and enzymatic mechanisms involved in the inactivation of conventional transmitters are not required for the inactivation of NO. Thus we believe we have satisfied the majority of the accepted criteria and that the most parsimonious conclusion from our experiments is that $\mathrm{NO}$ is the anterograde excitatory transmitter between the identified B2 and B7nor neurons. In support of this conclusion, our experiments on other transmitters known to be contained in B2 indicate that they are not mediators of the slow EPSP in B7nor.

At a systems level, our approach has exploited the advantages of the relatively simple organization of the CNS of the snail Lymnaea stagnalis that contains large, accessible, and uniquely identifiable neurons and well-characterized neural circuits with known behavioral roles. The best-characterized circuit in Lymnaea centrally generates the pattern of activity in motoneurons that underlies feeding behavior (Benjamin and Elliott, 1989). This CPG consists of an identified set of interneurons and is modulated by identified modulatory neurons such as the SO and the serotonergic cerebral giant cell (CGC). Evidence that NO is an important signaling molecule in feeding behavior comes from our earlier experiments showing that NO mediates the chemosensory activation of the feeding CPG (Elphick et al., 1995). These experiments did not identify the central neuronal targets for NO but clearly indicated that NO is a transmitter of chemosensory neurons that enter the CNS and have access to the 
neuronal components of the feeding CPG. We know now that NO is also a transmitter of central neurons that are part of the feeding CPG or are closely associated with it. One of these is the modulatory CGC that expresses both serotonin and a neuronal isoform of NOS (Korneev et al., 1998). In the present paper we identify the first NO-responsive neuron (B7nor) in the buccal ganglion that is involved in the feeding system and whose activity is entrained to feeding behavior. It is excited by the nitrergic B2 neuron, but our experiments do not exclude the possibility that it is also postsynaptic to other nitrergic neurons of the feeding system such as the CGC for example.

This novel type of a putative buccal neuron shows an activity pattern similar to that of previously described B7 motoneurons, i.e., excitation during protraction and strong inhibition during rasp (Benjamin and Rose, 1979; Staras et al., 1998). Unlike other B7 neurons, however, B7nor responds with a slow depolarization to B2 activity that is mediated by NO. During fictive feeding, B2 activity is weakly entrained to the on-going rhythm, and B2 activity mainly occurs during the rasp phase (Benjamin and Rose, 1979). The slow time course of the NO-mediated excitation from the B2 to B7nor ensures that the peak of the excitation occurs toward the end or just after the rasp phase. Therefore it is possible that the slow excitation aids the recovery of the B7nor neuron after its strong inhibition during the rasp phase. This demonstrates that NO transmission is involved in a neuronal circuit of known behavioral function, namely, feeding. With respect to this, it is of considerable interest that in Aplysia the identified C2 neuron, for which both histamine and NO are transmitters (Jacklet, 1995), excites a key modulatory neuron (the metacerebral cell) in the feeding system (Chiel et al., 1986).

A key observation in this paper is that NO can mediate slow excitatory synaptic transmission and possibly nonsynaptic transmission as well. Thus in some senses transmission by NO seems to be quite conventional. For example, it operates in the conventional anterograde direction from a presynaptic (B2) to a postsynaptic neuron (B7nor). Also, just as for conventional transmitters, the release of NO is coupled to the generation of action potentials in the presynaptic neuron and can cause unitary EPSPs in the follower neuron that are one-to-one with presynaptic impulses. The most straightforward explanation for this is that during the presynaptic action potential there is an influx of calcium that stimulates the synthesis of NO via the activation by a single action potential of a calcium- and calmodulin-regulated neuronal NOS (Korneev et al., 1998). This interpretation is supported by the observation that elevated extracellular calcium in the intact CNS can enhance transmission and enables the synapse to transmit one-to-one. Facilitation, in particular in the intact CNS, and summation would also appear to be features of anterograde NO transmission that are shared with more traditional transmitter systems.

Our experiments also point, however, to unconventional aspects of neurotransmission and raise a number of questions related to the spatial and temporal dynamics of NO signaling. In particular, our findings in cell culture provide the first direct evidence that NO can function as a nonsynaptic transmitter. When the two neurons are cocultured, transmission can occur without the need for contact, although this requires stronger stimulation of the presynaptic B2 neuron than is required when the neurons have grown extensive arborizations and multiple contacts. In the intact CNS, the arborizations of the B2 and B7nor neurons are in sufficiently close proximity that conventional synapses between them cannot be ruled out. There is however no evidence from our experiments on the intact CNS that transmission between them with conventional signaling molecules actually occurs. The B2 neuron that expresses NOS and releases $\mathrm{NO}$ also synthesizes more conventional transmitters such as acetylcholine and a number of neuropeptides (Santama et al., 1994; Perry et al., 1998). However, the depolarizing response in B7nor to B2 stimulation can be attributed to NO alone, raising the possibility that conventional synaptic specializations between the two neurons do not exist. If this were the case, NO may be released over the whole surface of the B2 neuron, able to affect the follower neuron both at points of close apposition and elsewhere.

Our experiments show clearly that NO can function as an anterograde excitatory signaling molecule in the CNS. Transmission is slow but can potentially operate over a broader spatial domain than that of other signaling molecules such as peptides that cannot pass through membranes and whose diffusion must therefore be limited to the extracellular compartment. Perhaps the most intriguing questions that remain to be answered about NO signaling relate to spatial and temporal dynamics and to the role of $\mathrm{NO}$ in the context of adaptive neural networks and behavior. Continued investigation at all levels-molecular, cellular, and behavioral-in tractable preparations such as the one we have developed will likely be required to elucidate fully the special roles of the NO signaling system in the nervous system.

\section{REFERENCES}

Bains JS, Ferguson AV (1997) Nitric oxide depolarizes type II paraventricular nucleus neurons in vitro. Neuroscience 79:149-159.

Benjamin PR, Elliott CJH (1989) Snail feeding oscillator: the central pattern generator and its control by modulatory interneurons. In: Neuronal and cellular oscillators (Jacklet JW, ed), pp 173-214. New York: Dekker.

Benjamin PR, Rose RM (1979) Central generation of bursting in the feeding system of the snail Lymnaea stagnalis. J Exp Biol 80:93-118.

Benjamin PR, Winlow W (1981) The distribution of three wide-acting synaptic inputs to identified neurons in the isolated brain of Lymnaea stagnalis (L). Comp Biochem Physiol (A) 70:293-307.

Benjamin PR, Rose RM, Slade CT, Lacy MG (1979) Morphology of identified neurons in the buccal ganglia of Lymnaea stagnalis. J Exp Biol 80:119-135.

Bredt DS, Snyder SH (1990) Isolation of nitric oxide synthetase, a calmodulin-requiring enzyme. Proc Natl Acad Sci USA 87:682-685.

Bredt DS, Snyder SH (1992) Nitric oxide, a novel neuronal messenger. Neuron 8:3-11.

Chiel H, Weiss K, Kupfermann I (1986) An identified histaminergic neuron modulates feeding motor circuitry in Aplysia. J Neurosci 6:2427-2450.

Dawson TM, Snyder SH (1994) Gases as biological messengers. Nitric oxide and carbon monoxide in the brain. J Neurosci 14:5147-5159.

Dobashi K, Pahan J, Chahal A, Singh I (1997) Modulation of endogenous antioxidant enzymes by nitric oxide in rat C6 glial cells. J Neurochem 68:1896-1903.

Elliott CJH, Benjamin PR (1985a) Interactions of pattern-generating interneurons controlling feeding in Lymnaea stagnalis. J Neurophysiol 54:1396-1411.

Elliott CJH, Benjamin PR (1985b) Interactions of the slow oscillator interneuron with feeding pattern-generating interneurons in Lymnaea stagnalis. J Neurophysiol 54:1412-1421.

Elphick MR, Kemenes G, Staras K, O’Shea M (1995) Behavioral role for nitric oxide in chemosensory activation of feeding in a mollusc. J Neurosci 15:7653-7664.

Garthwaite J, Boulton CL (1995) Nitric oxide signaling in the central nervous system. Annu Rev Physiol 57:683-706.

Garthwaite J, Charles SL, Chess-Williams R (1988) Endotheliumderived relaxing factor release on activation of NMDA receptors suggests role as intracellular messenger in the brain. Nature 336:385-388.

Gelperin A (1994) Nitric oxide mediates network oscillations of olfactory interneurons in a terrestrial mollusc. Nature 369:61-63. 
Getting PA (1981) Mechanisms of pattern generation underlying swimming in Tritonia. I. Neuronal network formed by monosynaptic connections. J Neurophysiol 46:65-79.

Grozdanovic Z, Nakos G, Mayer B, Gossrau R (1995) A modified method allows for correlation between NADH-diaphorase histochemistry and immunohistochemistry for the demonstration of neuronal nitric oxide synthase (nNOS). Folia Histochemica et Cytobiologica $33: 11-18$.

Hartell NA (1996) Strong activation of parallel fibers produces localized calcium transients and a form of LTD that spreads to distant synapses. Neuron 16:601-610.

Hibbs JB, Vavrin Z, Taintor RR (1987) L-Arginine is required for expression of the activated macrophage effector mechanism causing selective metabolic inhibition in target cells. J Immunol 138:550-565.

Hölscher C (1997) Nitric oxide, the enigmatic neuronal messenger: its role in synaptic plasticity. Trends Neurosci 20:298-303.

Jacklet J (1995) Nitric oxide is used as an orthograde cotransmitter at identified histaminergic synapses. J Neurophysiol 74:891-895.

Kogan VB, Fridman MT, Kafaov W (1963) In: Solubilities of inorganic and organic compounds, Vol 1 (Stephen H, Stephen T, eds), p 330. New York: Pergamon.

Korneev SA, Piper MR, Picot J, Phillips R, Korneeva EI, O'Shea M (1998) Molecular characterization of NOS in a mollusc: expression in a giant modulatory neuron. J Neurobiol 35:65-76.

Lancaster J (1994) Simulation of the diffusion and reaction of endogenously produced nitric oxide. Proc Natl Acad Sci USA 91:8137-8141.

Lei SZ, Pan Z-H, Aggarwal SK, Chen H-S, Harman J, Sucher N, Lipton SA (1992) Effect of nitric oxide production on the redox modulatory site of the NMDA receptor-channel complex. Neuron 8:1087-1099.

Li J, Smith SS, McElliott JG (1995) Cerebellar nitric oxide is necessary for vestibulo-ocular reflex adaptation, a sensorimotor model of learning. J Neurophysiol 74:489-494.

Moroz LL, Bulloch AGM, Lukowiak K, Syed NI (1994a) Putative NOSsynthesizing neurons of Lymnaea in vivo and in vitro. Netherlands $\mathrm{J}$ Zool 44:535-549.

Moroz LL, Winlow W, Turner RW, Bulloch AGM, Lukowiak K, Syed NI (1994b) Nitric oxide synthase-immunoreactive cells in the CNS and periphery of Lymnaea. NeuroReport 5:1277-1280.

O'Shea M, Colbert R, Williams L, Dunn S (1998) Nitric oxide compartments in the mushroom bodies of the locust brain. NeuroReport 9:333-336.
Park JH, Budko DY, Moroz LL, Winlow W (1995) Optical monitoring of movements in small animals and in semi-intact preparations. J Neurosci Methods 56:181-186.

Perry SJ, Straub VA, Kemenes G, Santama N, Worster BM, Burke JF, Benjamin PR (1998) Neural modulation of gut motility by the myomodulin peptides and acetylcholine in the snail Lymnaea. J Neurophysiol 79:2460-2474.

Ridgeway RL, Syed NI, Lukowiak K, Bulloch AG (1991) Nerve growth factor (NGF) induces sprouting of specific neurons of the snail Lymnaea stagnalis. J Neurobiol 22:377-390.

Rose RM, Benjamin PR (1979) The relationship of the central motor pattern to the feeding cycle of Lymnaea stagnalis. J Exp Biol 80:137-163.

Rose RM, Benjamin PR (1981) Interneuronal control of feeding in the pond snail Lymnaea stagnalis. I. Initiation of feeding cycles by a single buccal interneuron. J Exp Biol 92:187-201.

Santama N, Brierley M, Burke J, Benjamin P (1994) Neural network controlling feeding in Lymnaea stagnalis: immunocytochemical localization of myomodulin, small cardioactive peptide, buccalin and FMRFamide-related peptides. J Comp Neurol 343:352-365.

Staras K, Kemenes G, Benjamin PR (1998) Pattern-generating role for motoneurons in a rhythmically active neuronal network. J Neurosci 18:3669-3688.

Teyke T (1996) Nitric oxide, but not serotonin, is involved in acquisition of food-attraction conditioning in the snail Helix pomatia. Neurosci Lett 206:29-32.

Weiss KR, Shapiro E, Kupfermann I (1986) Modulatory synaptic actions of an identified histaminergic neuron on the serotonergic metacerebral cell of Aplysia. J Neurosci 6:2393-2402.

Winlow W (1987) Action potential plasticity. In: Growth and plasticity of neural connections (Winlow W, McCrohan CR, eds), pp 127-144. Manchester, United Kingdom: Manchester University.

Wood J, Garthwaite J (1994) Model of the diffusional spread of nitric oxide-implications for neural nitric oxide signaling and its pharmacological properties. Neuropharmacology 33:1235-1244.

Yeoman MS, Parish DC, Benjamin PR (1993) A cholinergic modulatory interneuron in the feeding system of the snail Lymnaea. J Neurophysiol $70: 37-50$. 